\title{
Alkynylation of Pentose Derivatives with Stereochemical Fidelity: Implications for the Regioselectivity of Alkynyl Diol Cycloisomerizations to Cyclic Enol Ethers
}

\author{
Frank E. McDonald, ${ }^{*}$ Dian Ding, Andrew J. Ephron, ${ }^{\dagger}$ and John Bacsa \\ Department of Chemistry, Emory University, Atlanta, Georgia 30322, United States \\ e-mail: fmcdona@emory.edu
}

SUPPORTING INFORMATION: ${ }^{1} \mathrm{H}$ and ${ }^{13} \mathrm{C}$ NMR spectra, part 2

2,3-benzylidene-protected ribofuranose 5

$\begin{array}{ll}\text { Alkynyl diol } 7 & 34\end{array}$

D-arabino-septanose glycal $8 \quad 37$

2,3-benzylidene-protected lyxofuranose 10a and 10b 39

Alkynyl diols 11a and 11b 41

Septanose glycals 12a and 12b $\quad 47$

$\begin{array}{ll}\text { Alkynyl diol } \mathbf{1 4} & 51\end{array}$

Alkynyl diols 15a and 15b $\quad 55$

Pyranose glycal $16 \quad 63$

Pyranose glycals 17a and 17b 66 
2,3-Benzylidene-protected ribofuranose, 5, ${ }^{1} \mathrm{H} \mathrm{NMR}\left(400 \mathrm{MHz}, \mathrm{CDCl}_{3}\right)$

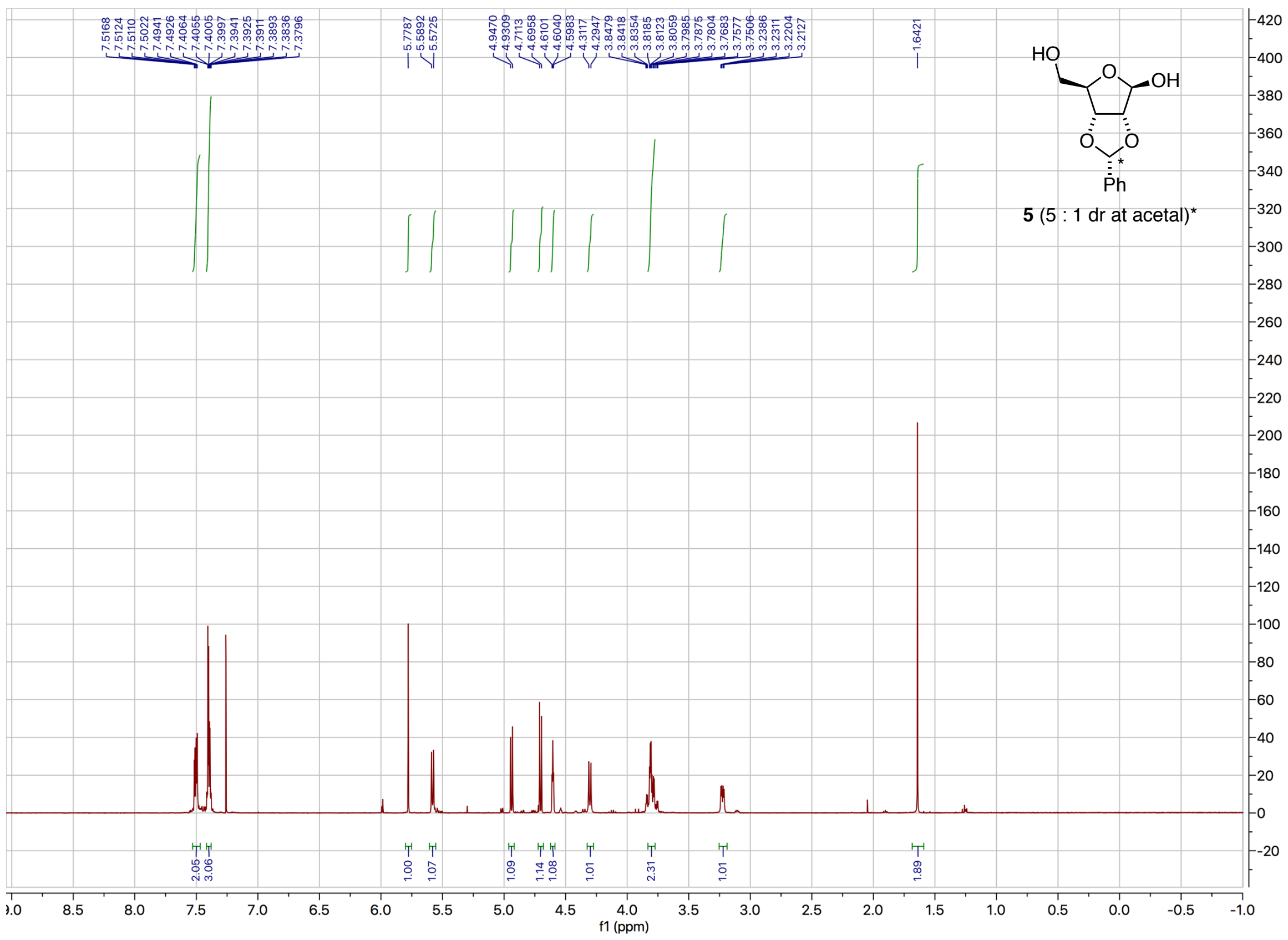


2,3-Benzylidene-protected ribofuranose, 5, ${ }^{13} \mathrm{C} \mathrm{NMR} \mathrm{(126} \mathrm{MHz,} \mathrm{CDCl}_{3}$ )

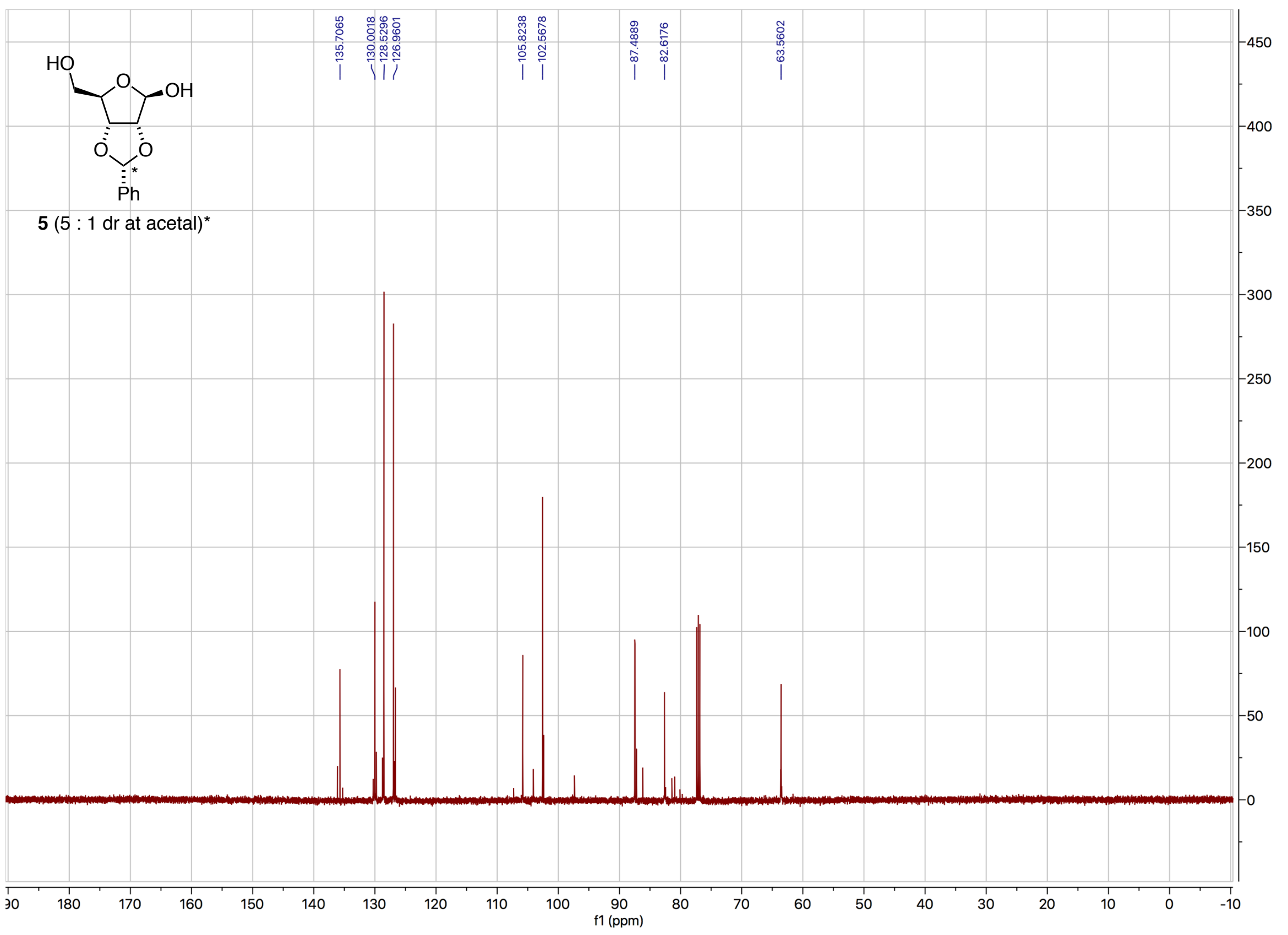

S-33 
Alkynyl diol 7, ${ }^{1} \mathrm{H}$ NMR (400 MHz, $\left.\mathrm{CDCl}_{3}\right)$

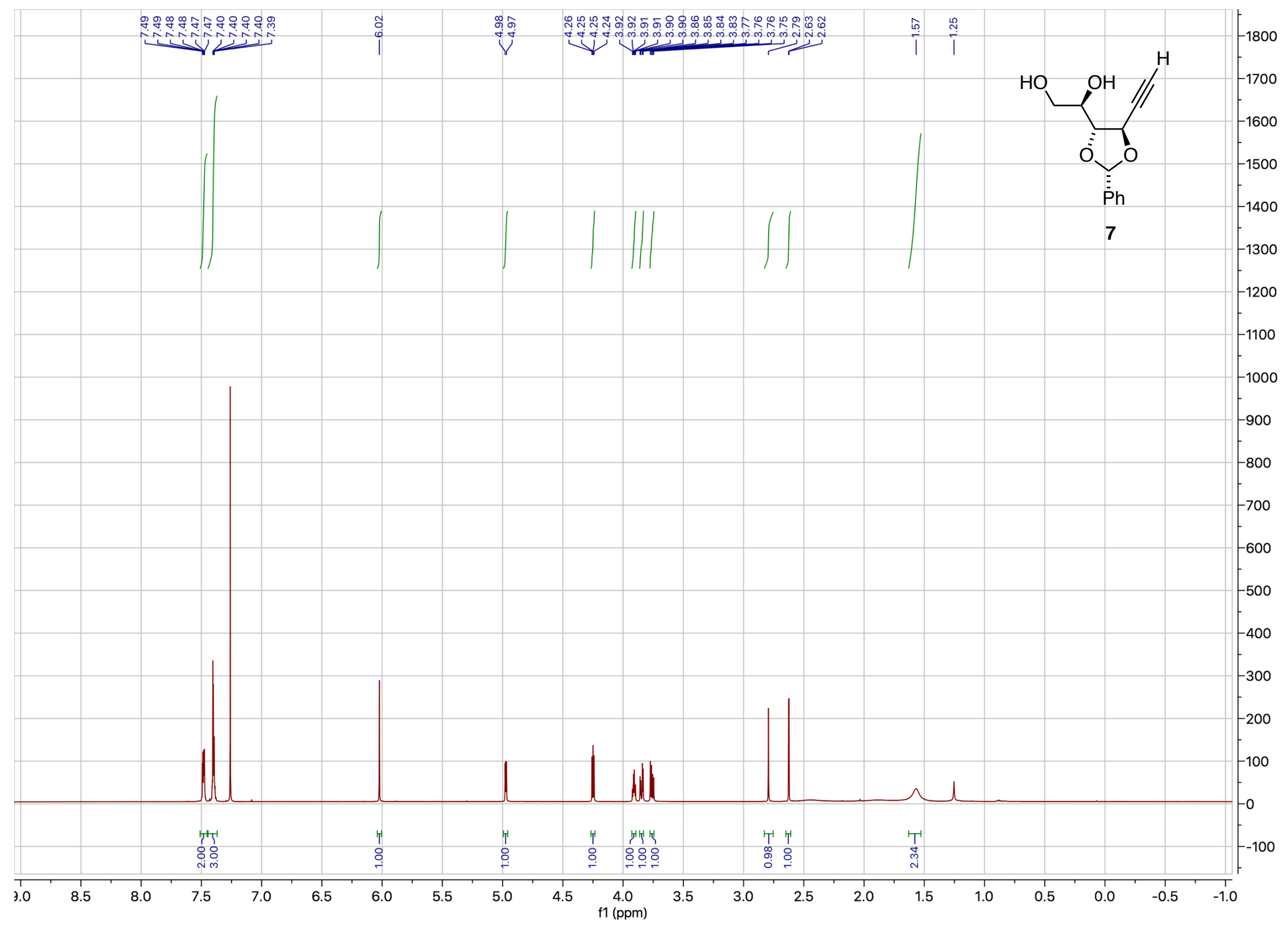

S-34 
Alkynyl diol 7, ${ }^{1} \mathrm{H}$ NMR (expansion)

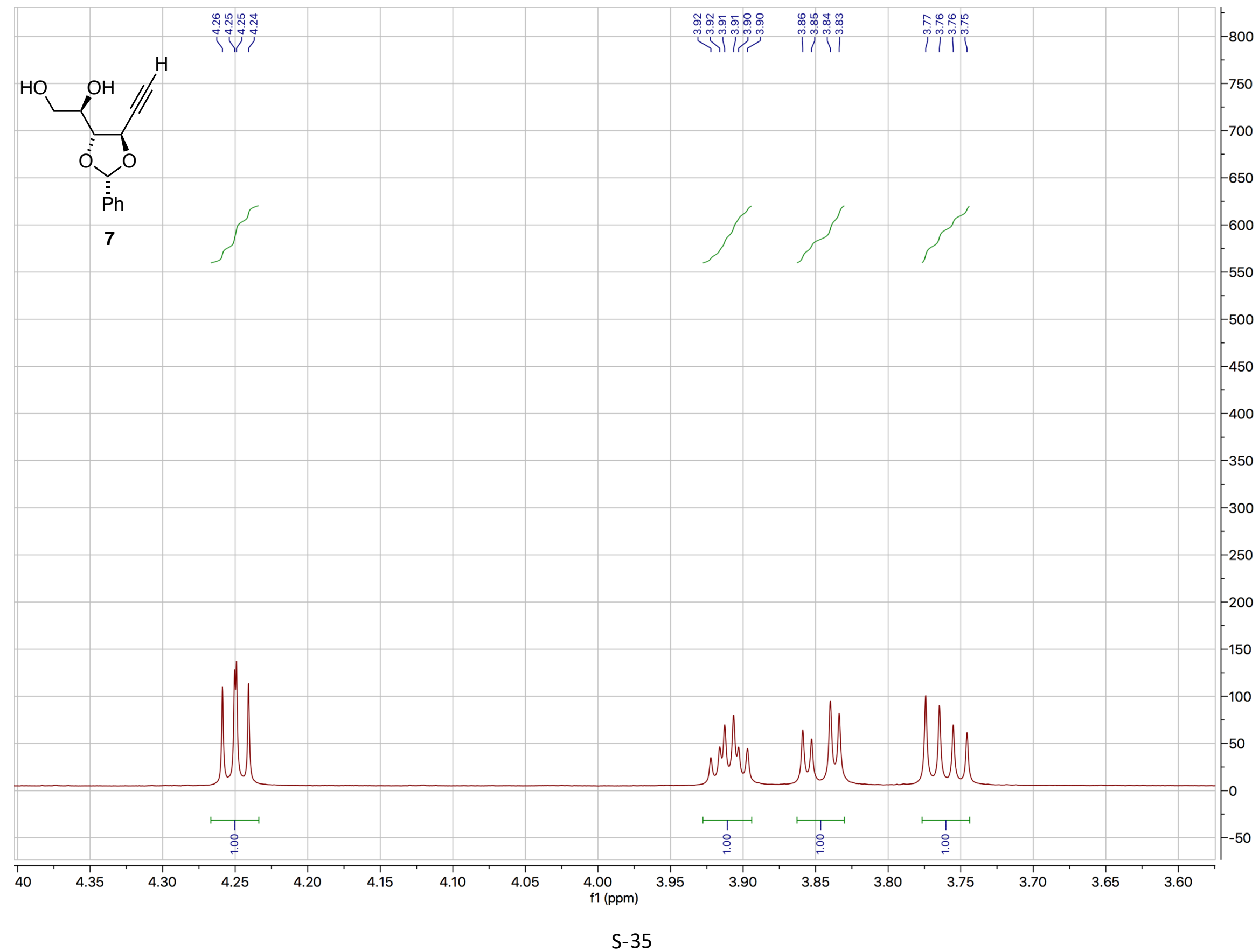


Alkynyl diol 7, ${ }^{13} \mathrm{C}$ NMR (151 MHz, $\mathrm{CDCl}_{3}$ )

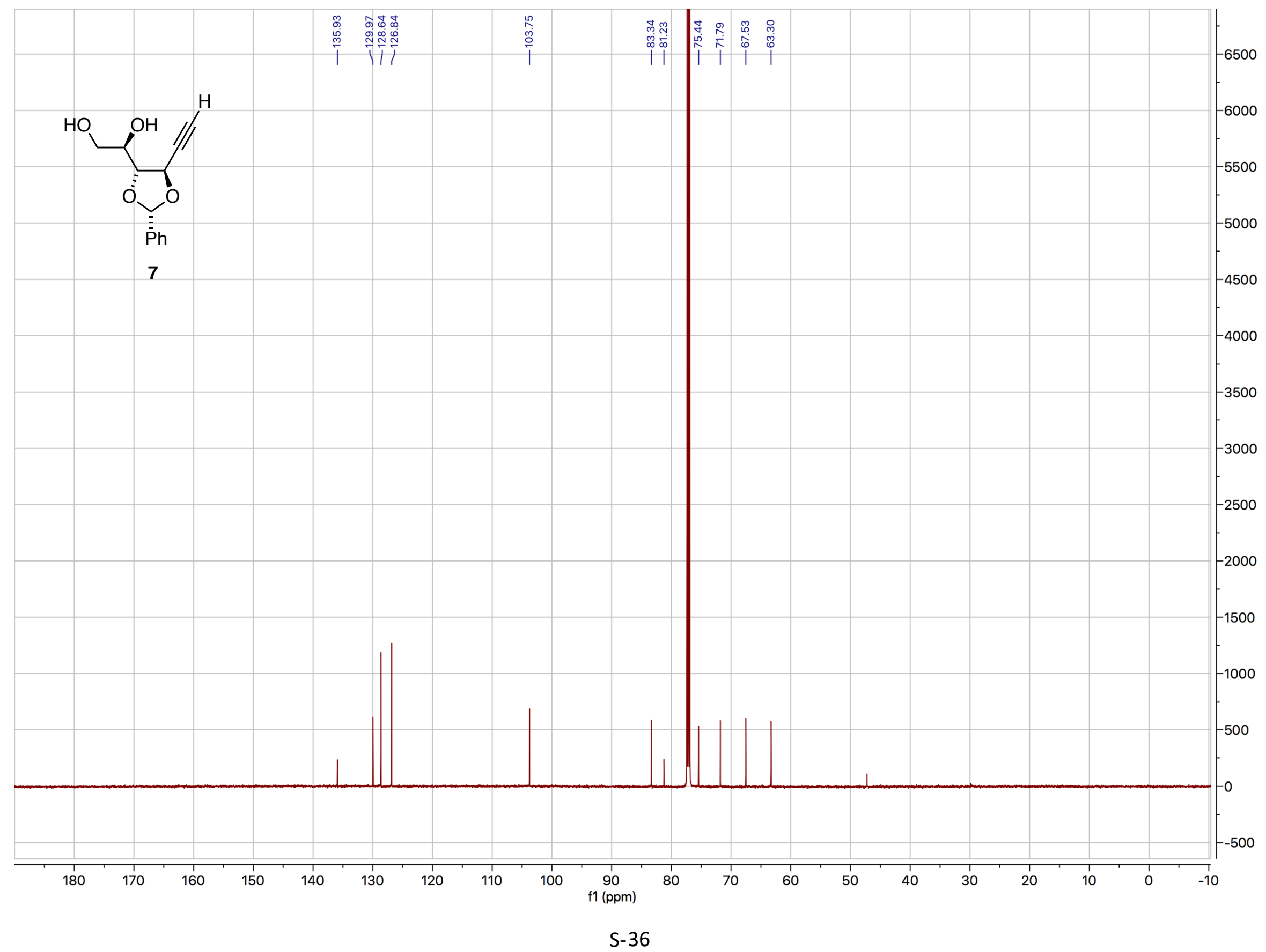


Septanose glycal 8, ${ }^{1} \mathrm{H}$ NMR (500 MHz, $\left.\mathrm{CDCl}_{3}\right)$

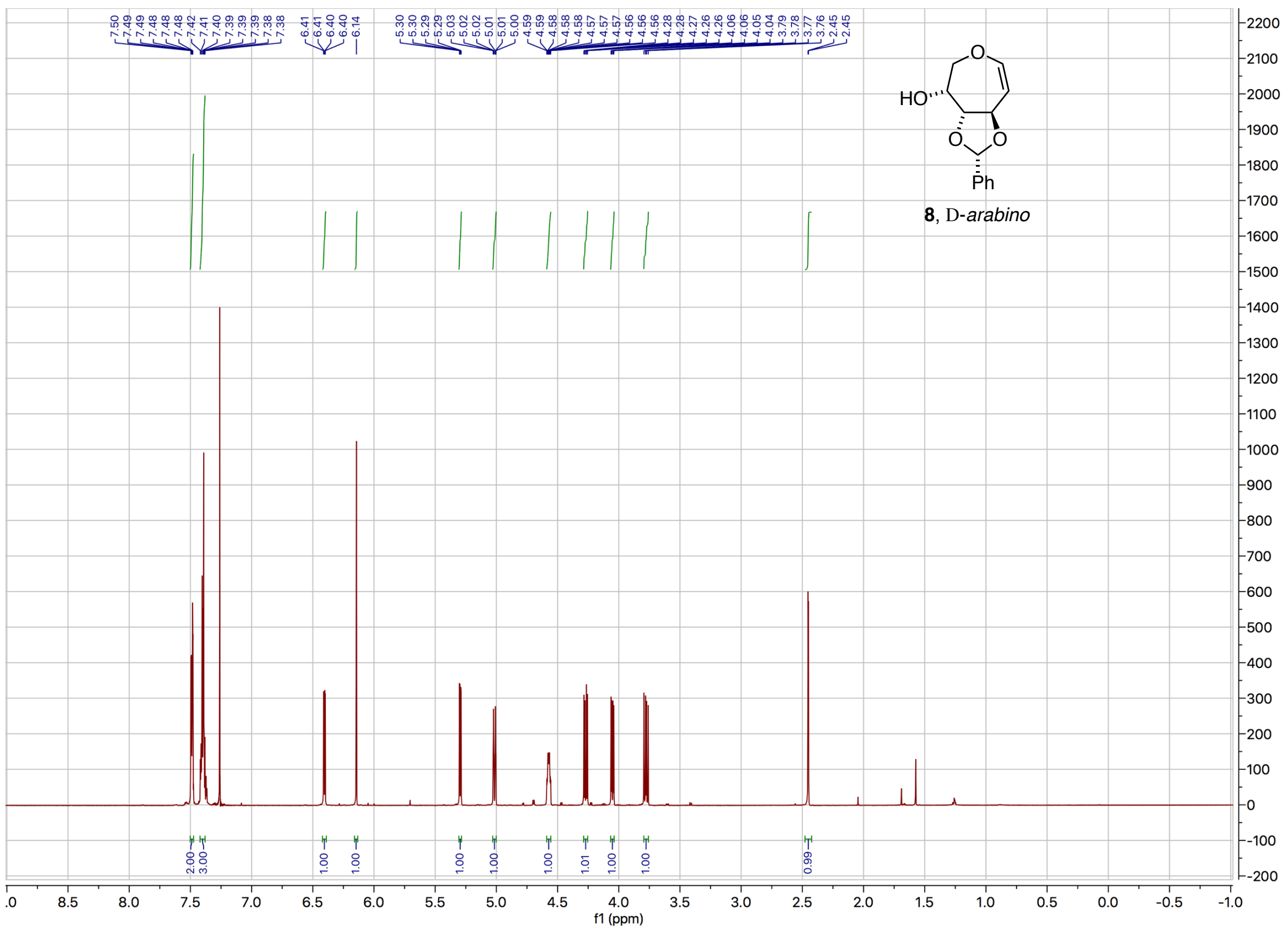


Septanose glycal 8, ${ }^{13} \mathrm{C}$ NMR (126 $\left.\mathrm{MHz}, \mathrm{CDCl}_{3}\right)$

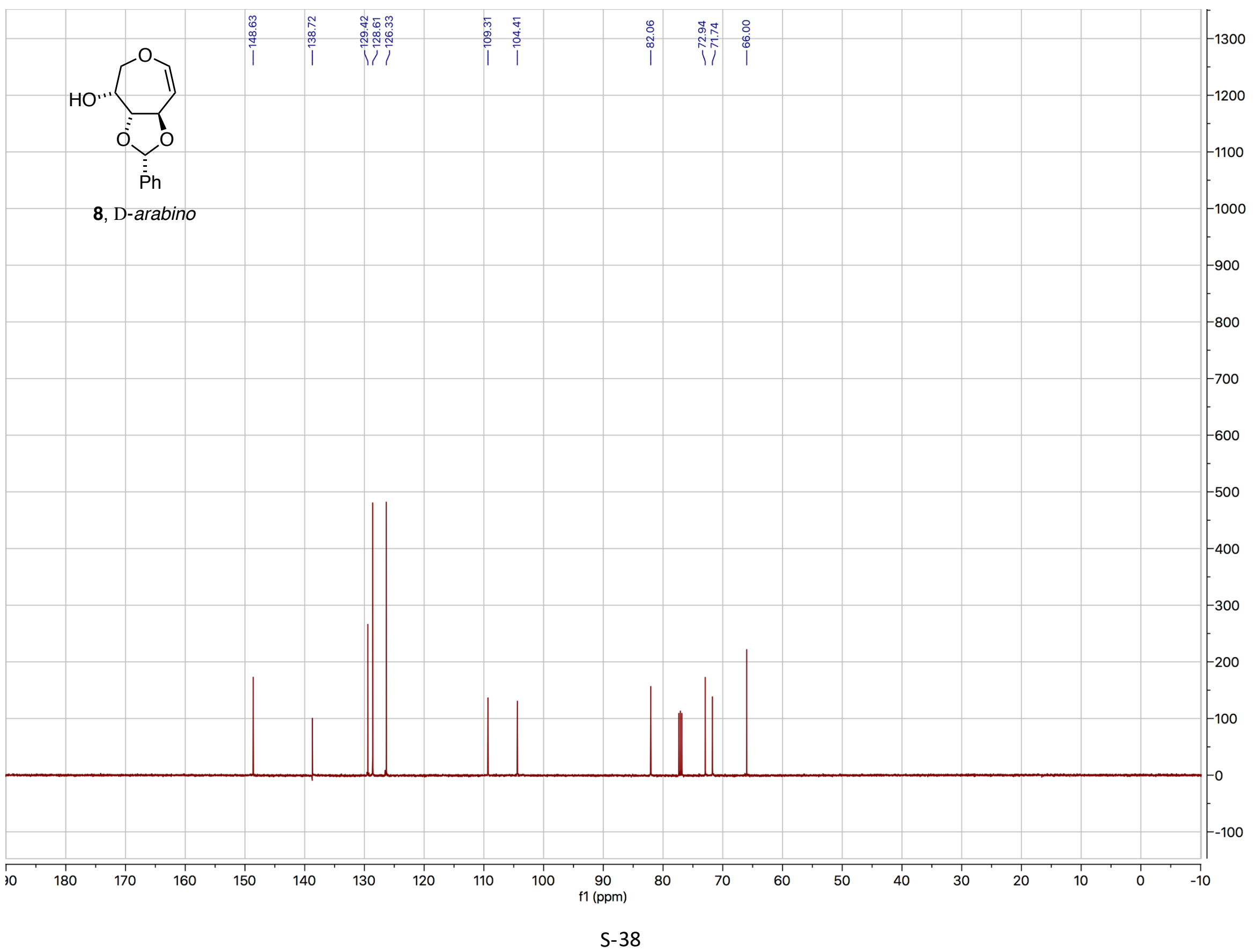


2,3-Benzylidene-protected lyxofuranose, 10a-10b, ${ }^{1} \mathrm{H}$ NMR (400 MHz, DMSO- $\left.d_{6}\right)$

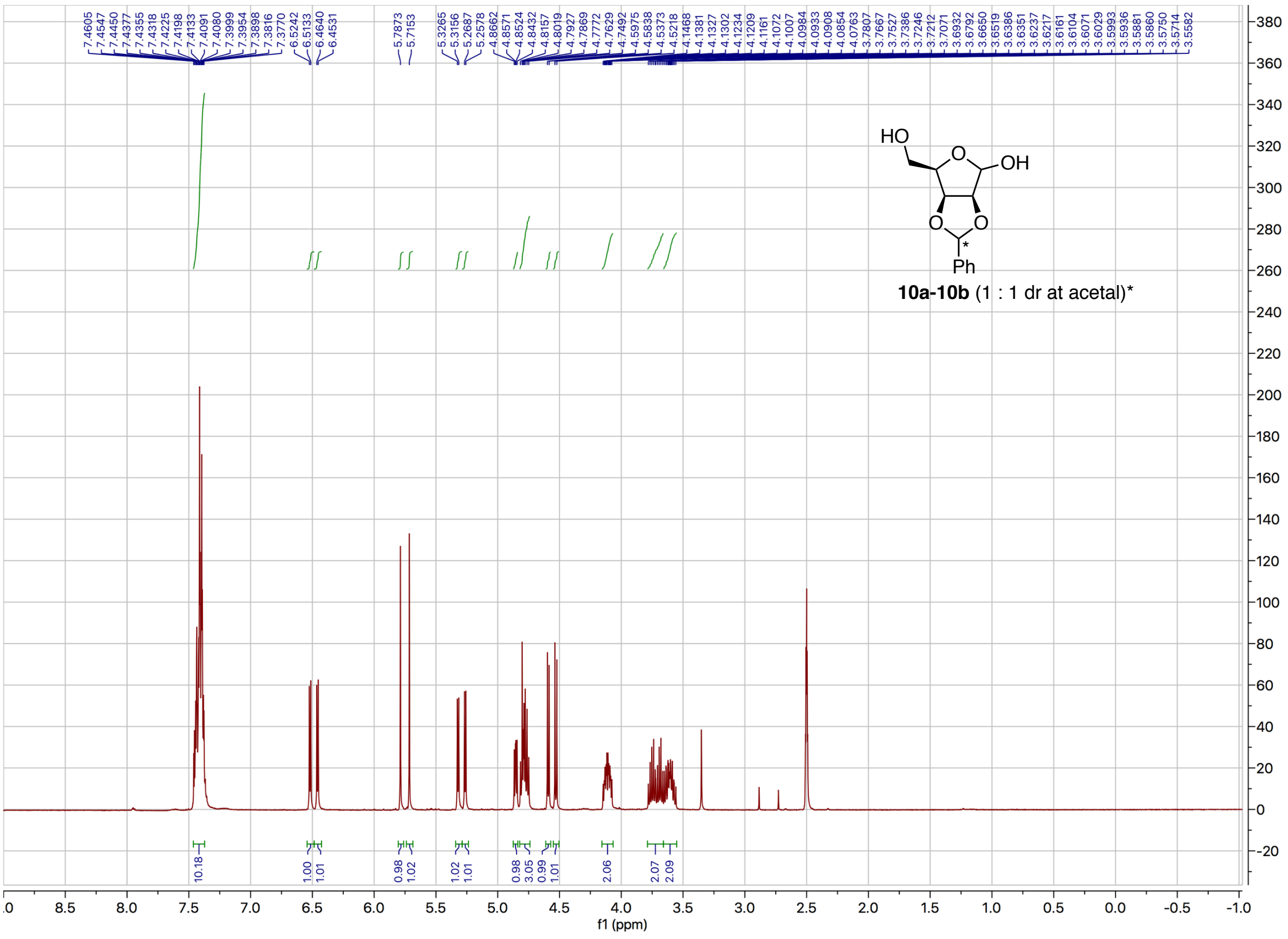


2,3-Benzylidene-protected lyxofuranose, 10a-10b, $\left.{ }^{13} \mathrm{C} \mathrm{NMR} \mathrm{(126} \mathrm{MHz}, \mathrm{CDCl}_{3}\right)$

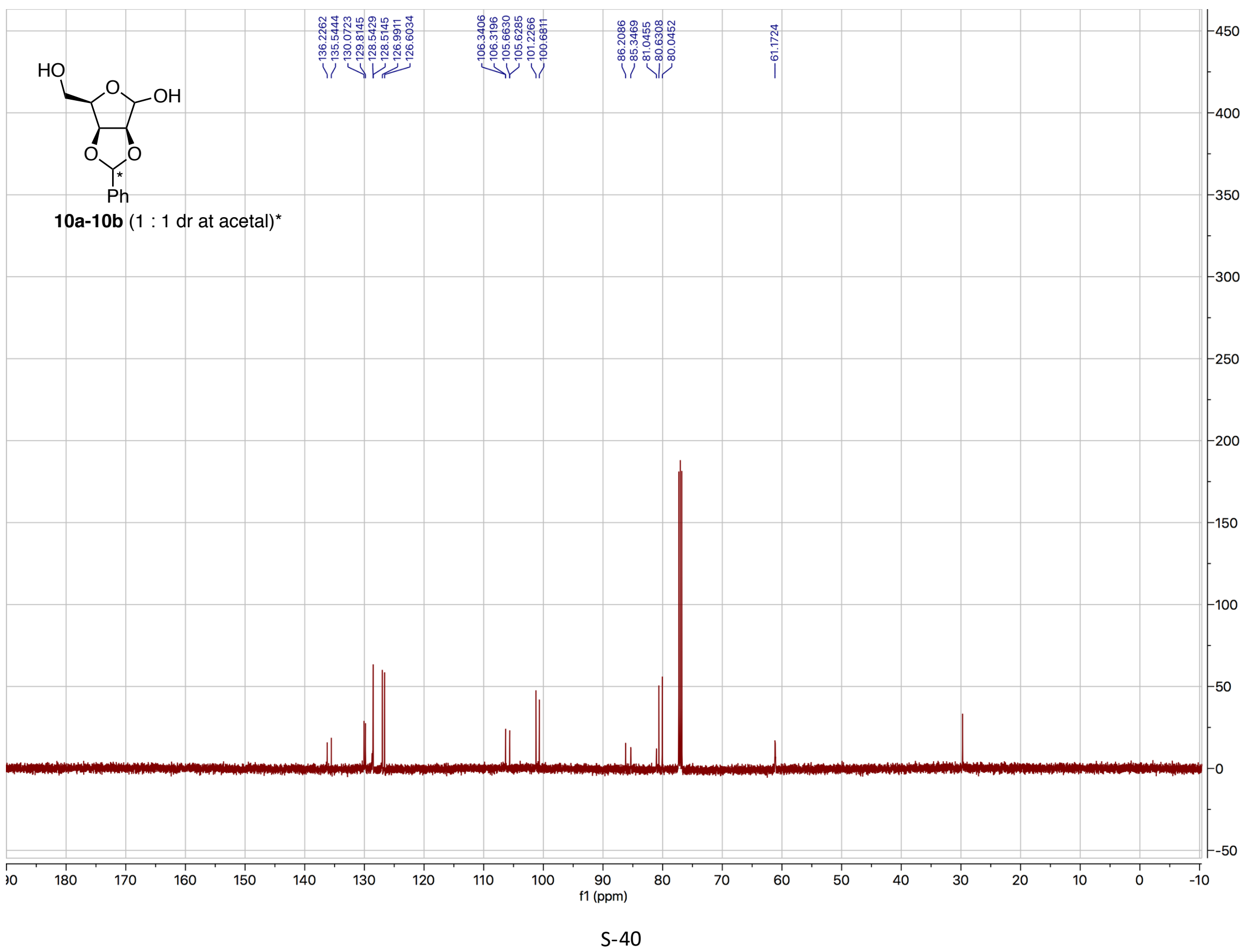


Alkynyl diol 11a, ${ }^{1} \mathrm{H}$ NMR (600 MHz, $\left.\mathrm{CDCl}_{3}\right)$

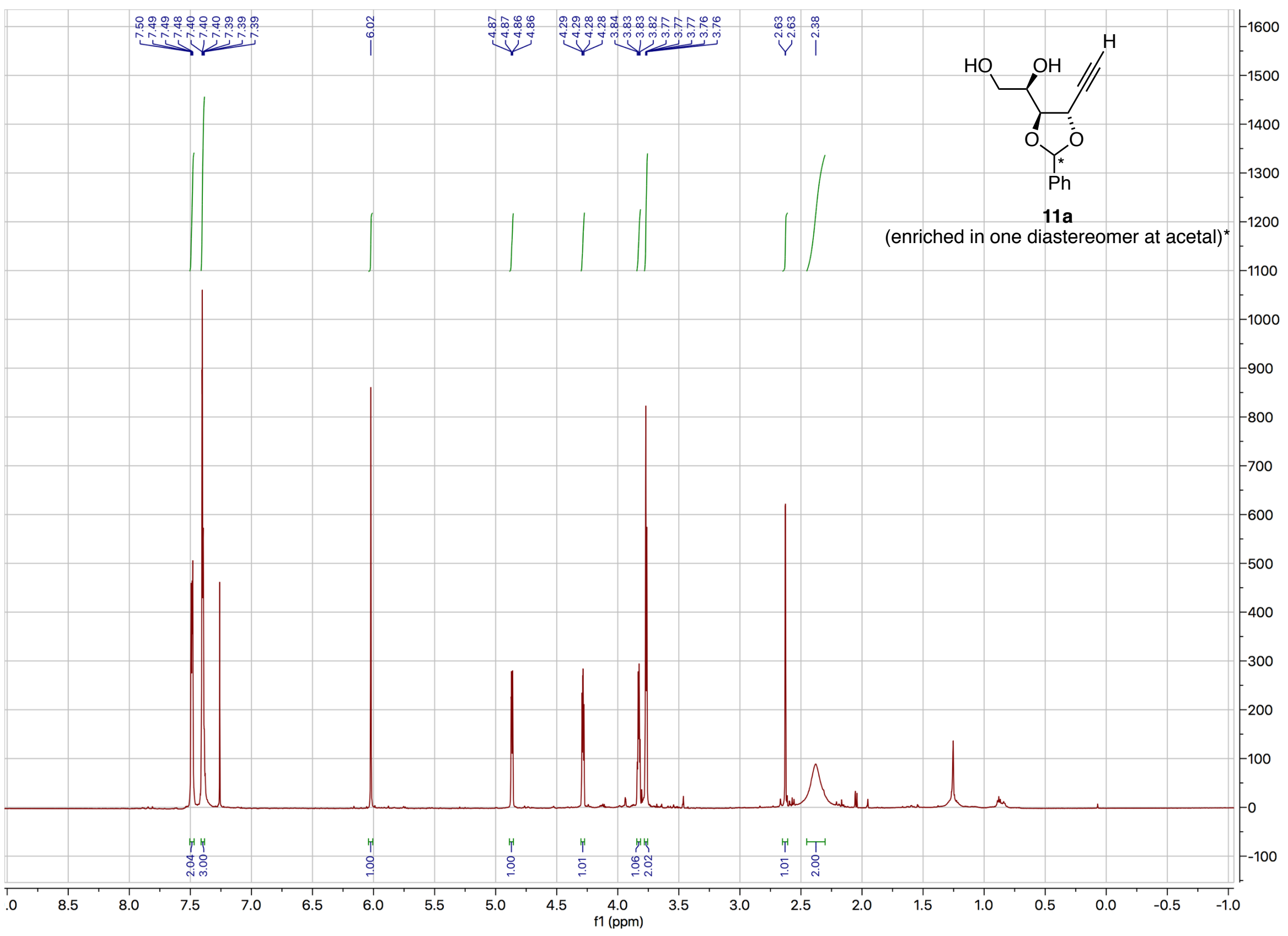

S-41 
Alkynyl diol 11a, ${ }^{1} \mathrm{H}$ NMR (expansion)




Alkynyl diol 11a, ${ }^{13} \mathrm{C}$ NMR (151 MHz, $\left.\mathrm{CDCl}_{3}\right)$

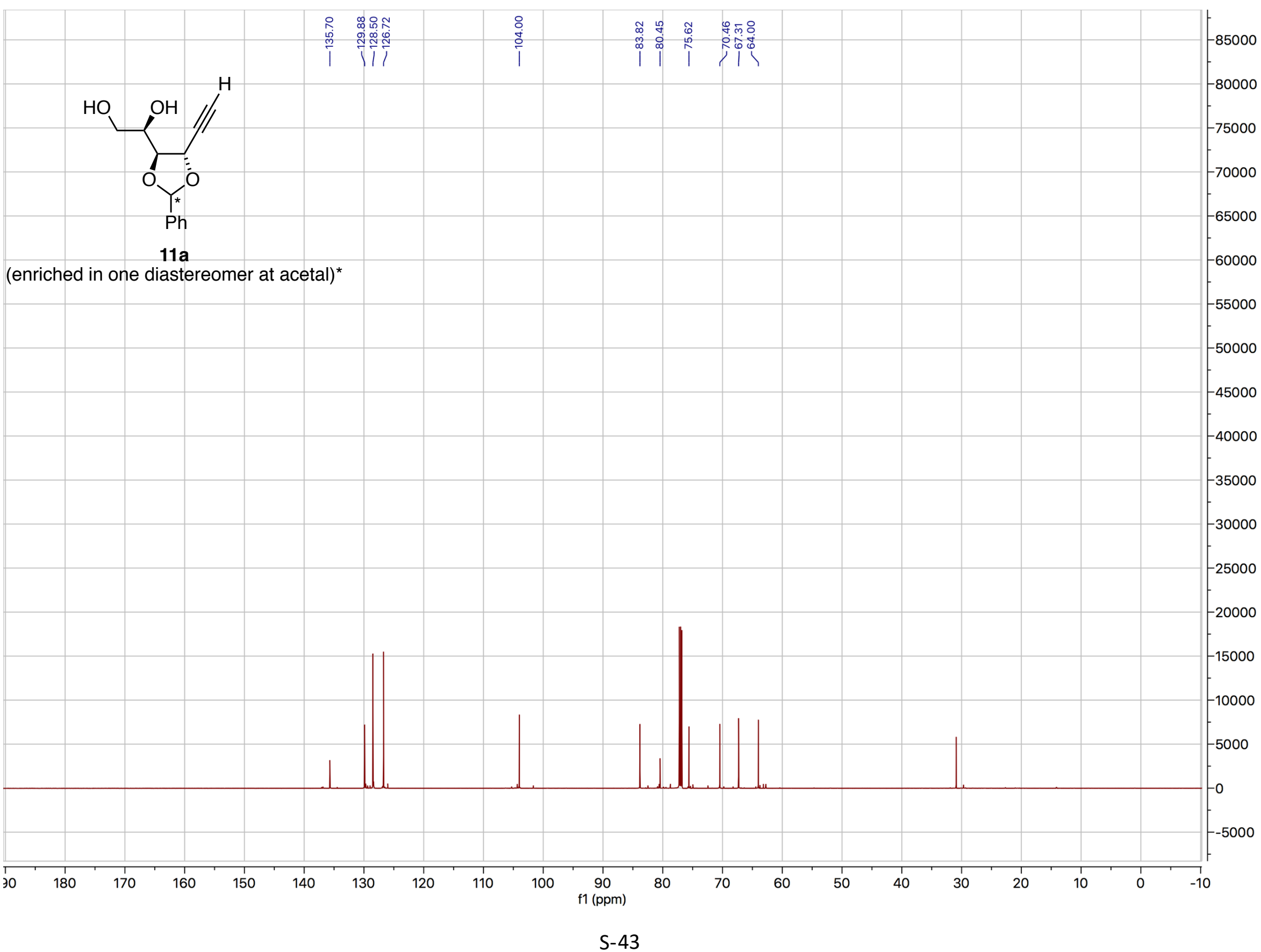


Alkynyl diol 11b, ${ }^{1} \mathrm{H}$ NMR (600 MHz, $\left.\mathrm{CDCl}_{3}\right)$

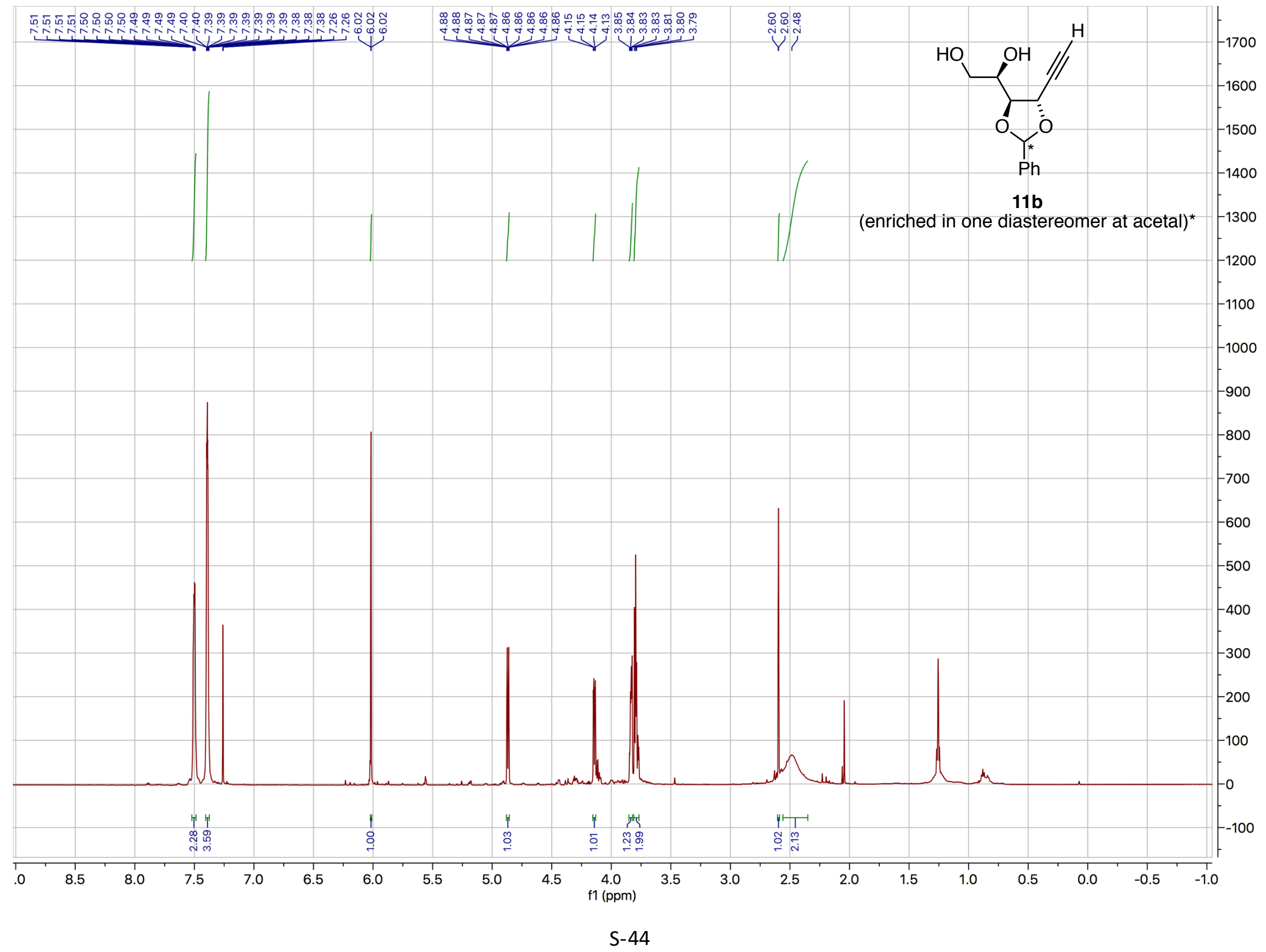


Alkynyl diol 11b, ${ }^{1} \mathrm{H}$ NMR (expansion)

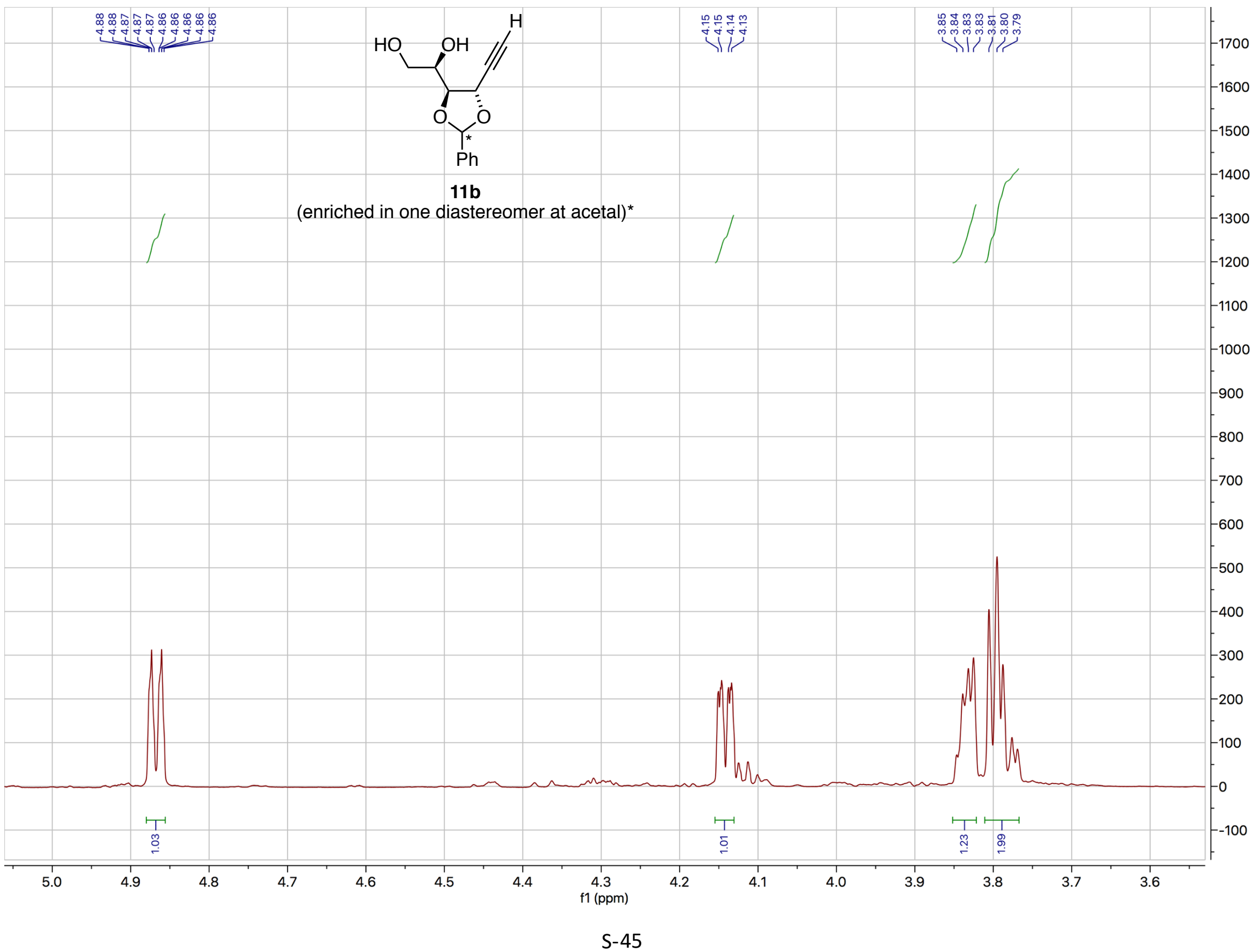


Alkynyl diol 11b, ${ }^{13} \mathrm{C}$ NMR (151 MHz, $\left.\mathrm{CDCl}_{3}\right)$

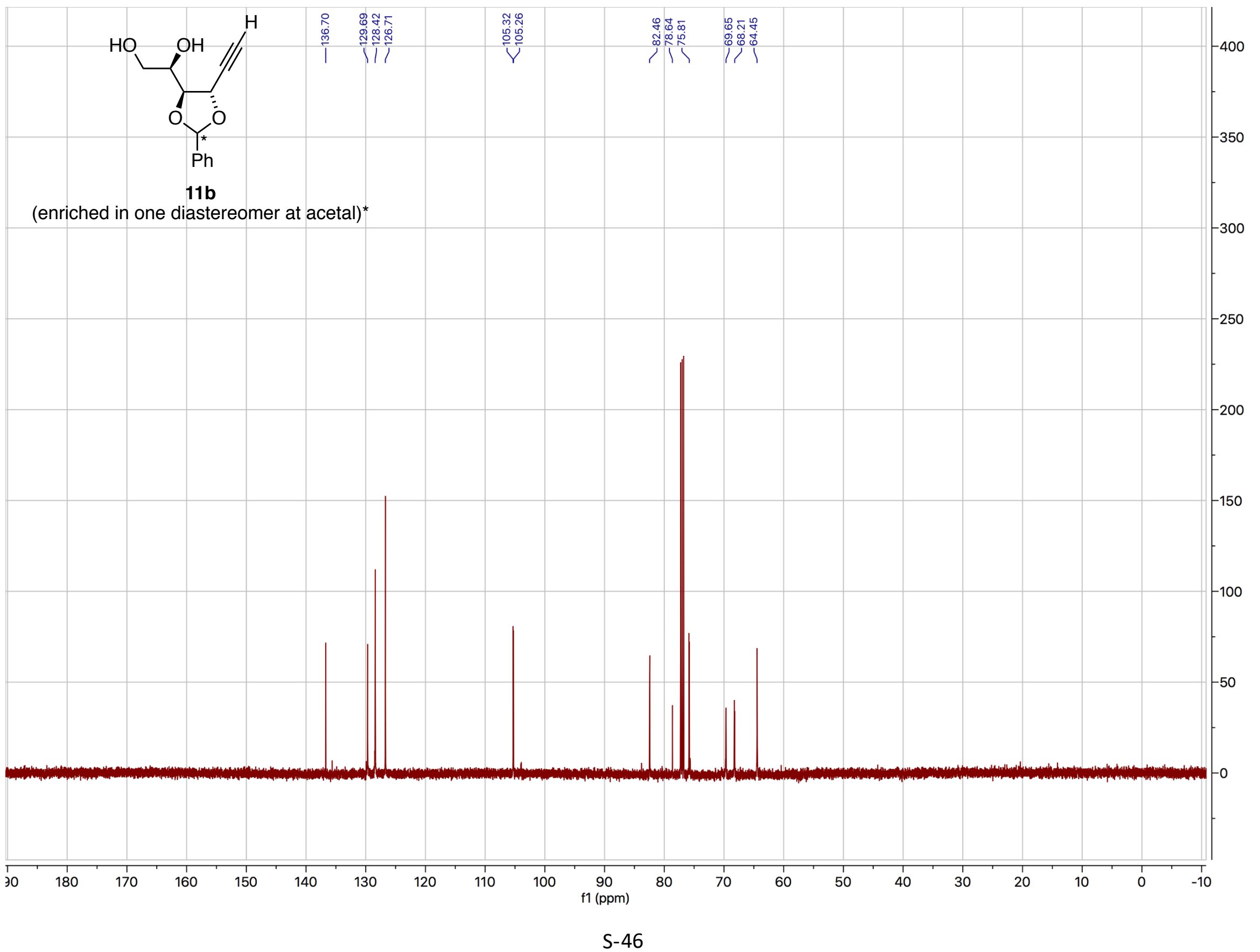


Septanose glycal 12a, ${ }^{1} \mathrm{H}$ NMR $\left(400 \mathrm{MHz}, \mathrm{CDCl}_{3}\right)$

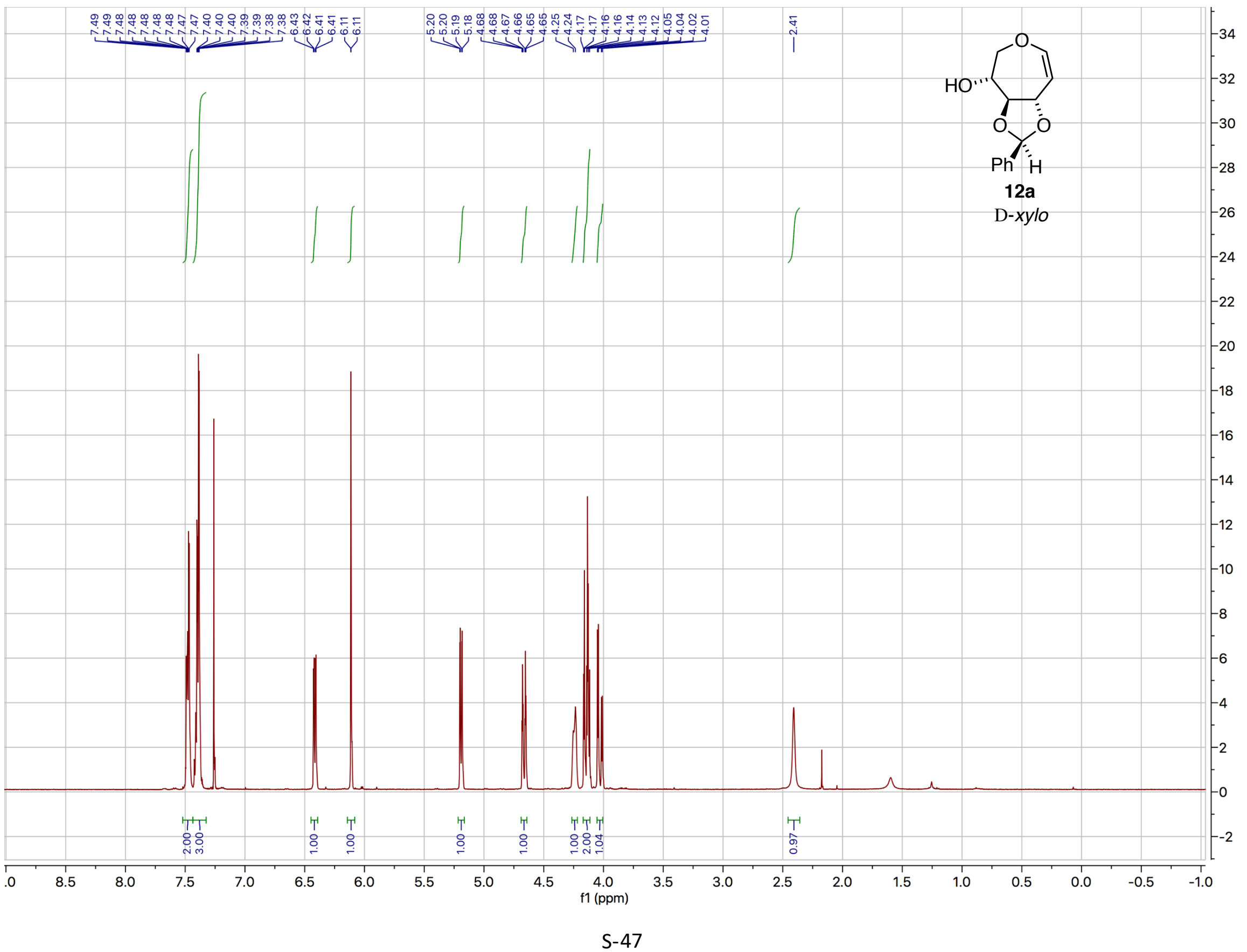


Septanose glycal 12a, ${ }^{13} \mathrm{C}$ NMR (126 MHz, $\left.\mathrm{CDCl}_{3}\right)$

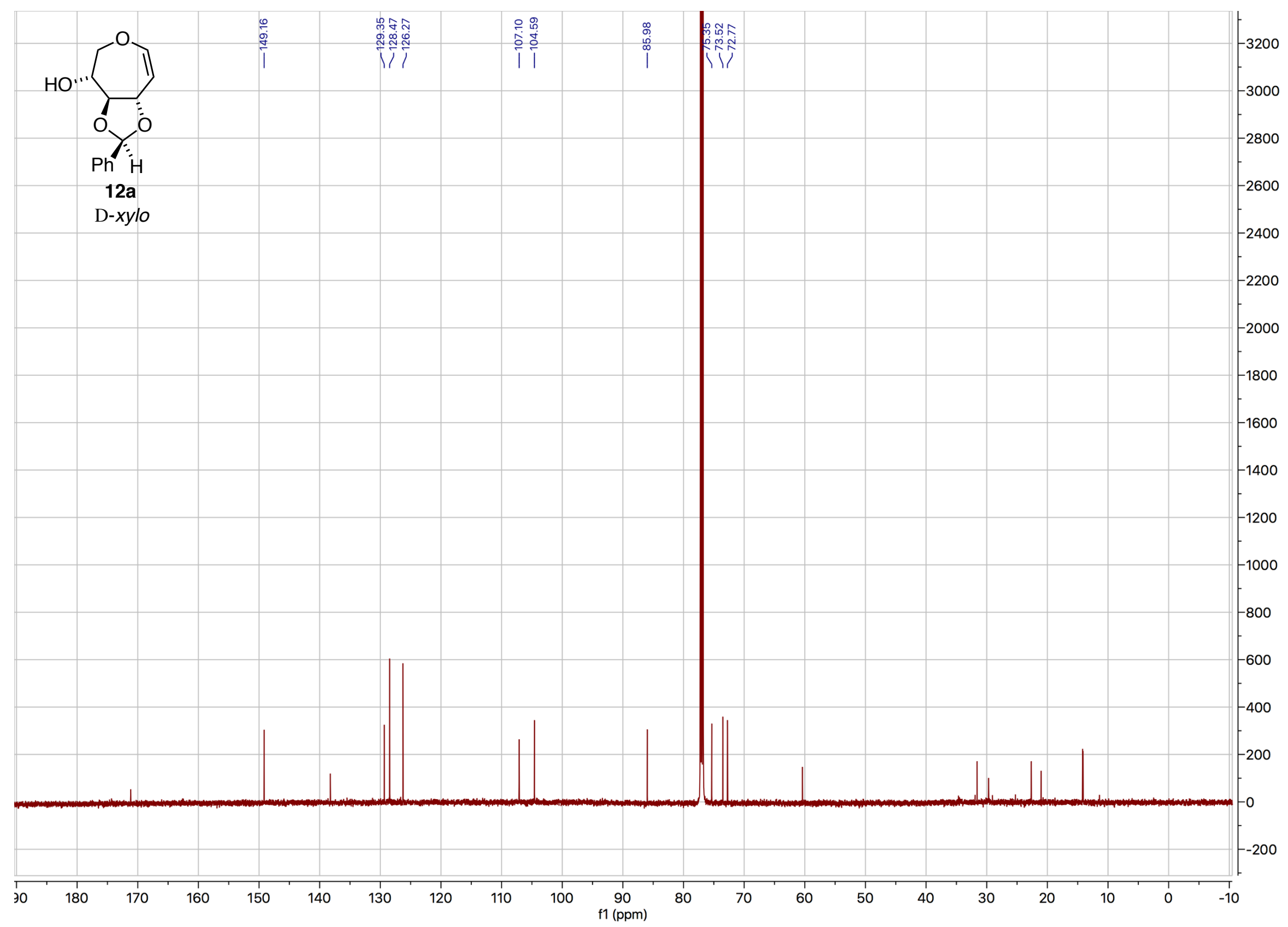

S-48 
Septanose glycal 12b, ${ }^{1} \mathrm{H}$ NMR (399 MHz, $\left.\mathrm{CDCl}_{3}\right)$

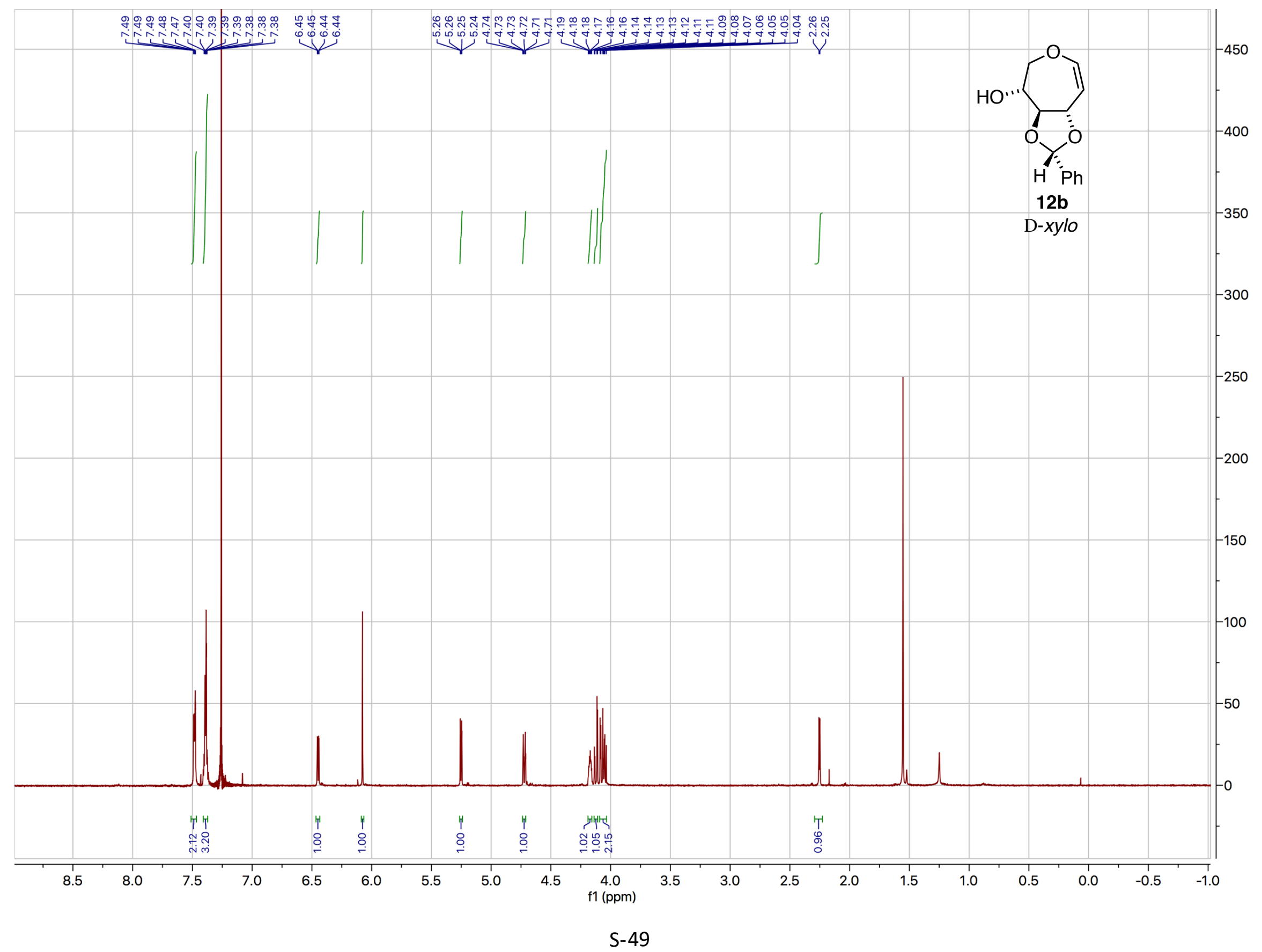


Septanose glycal 12b, ${ }^{13} \mathrm{C}$ NMR (126 MHz, $\mathrm{CDCl}_{3}$ )

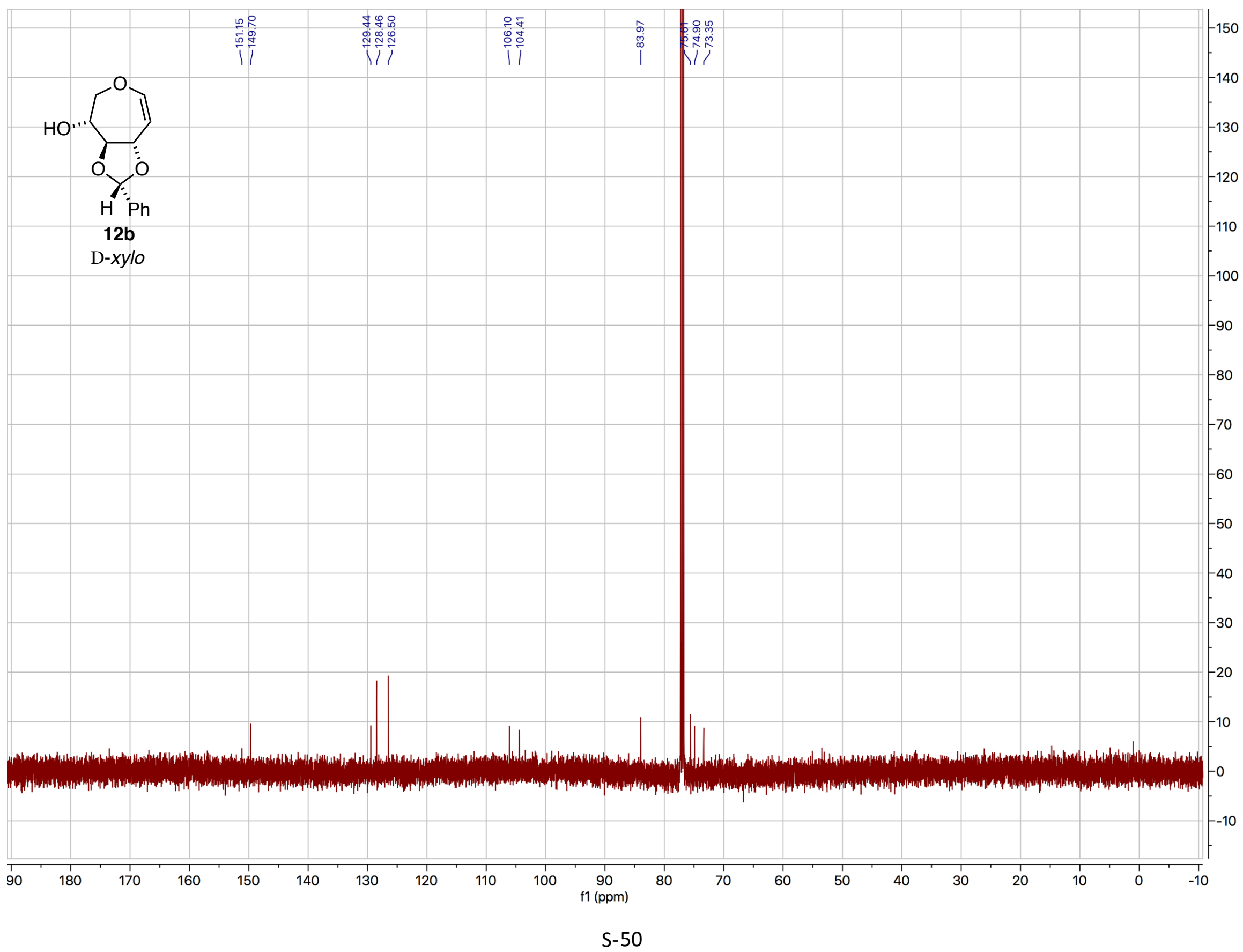


Alkynyl diol 14, ${ }^{1} \mathrm{H}$ NMR (400 MHz, $\mathrm{CDCl}_{3}$ )

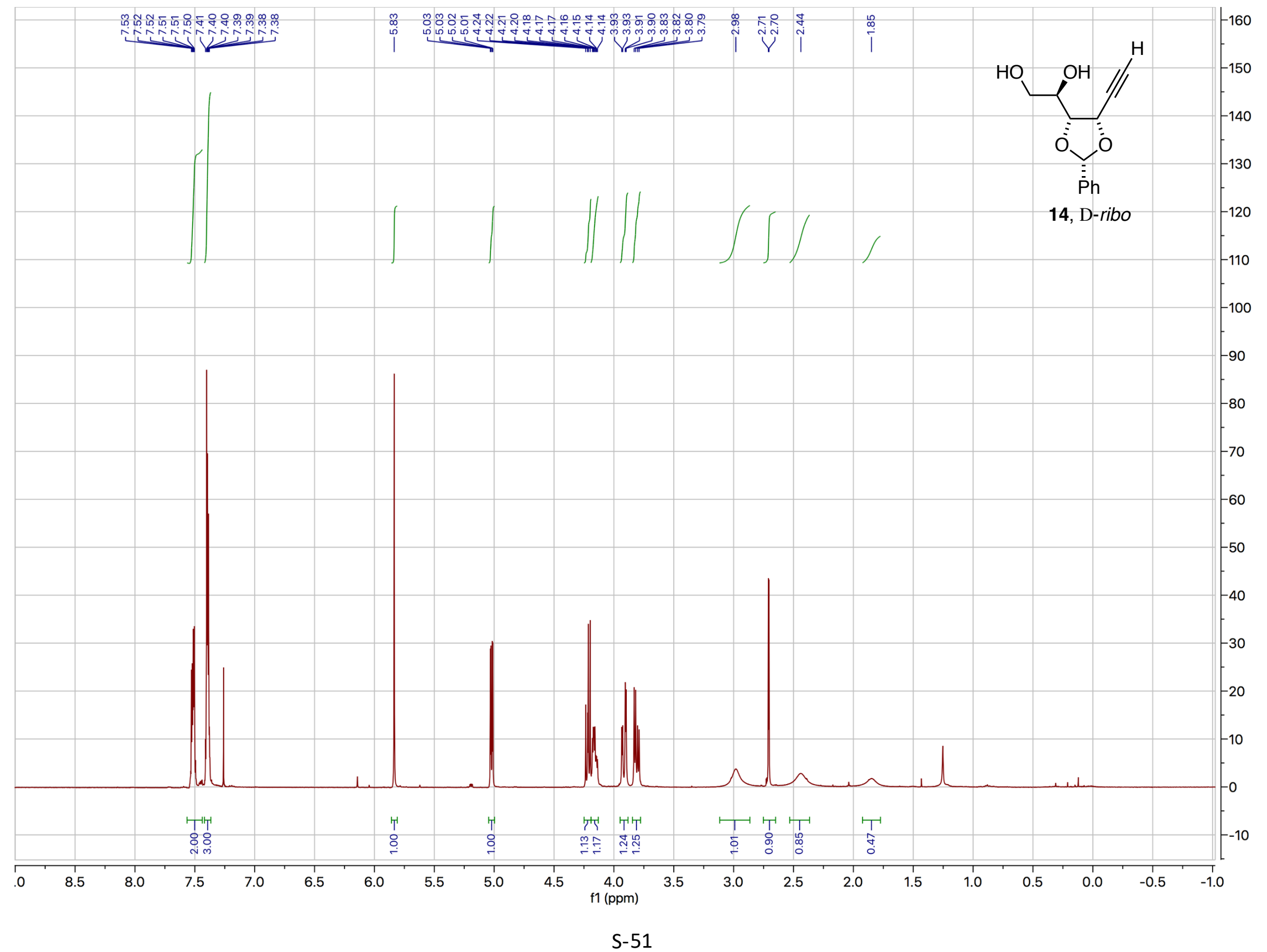


Alkynyl diol 14, ${ }^{1} \mathrm{H}$ NMR (expansion)

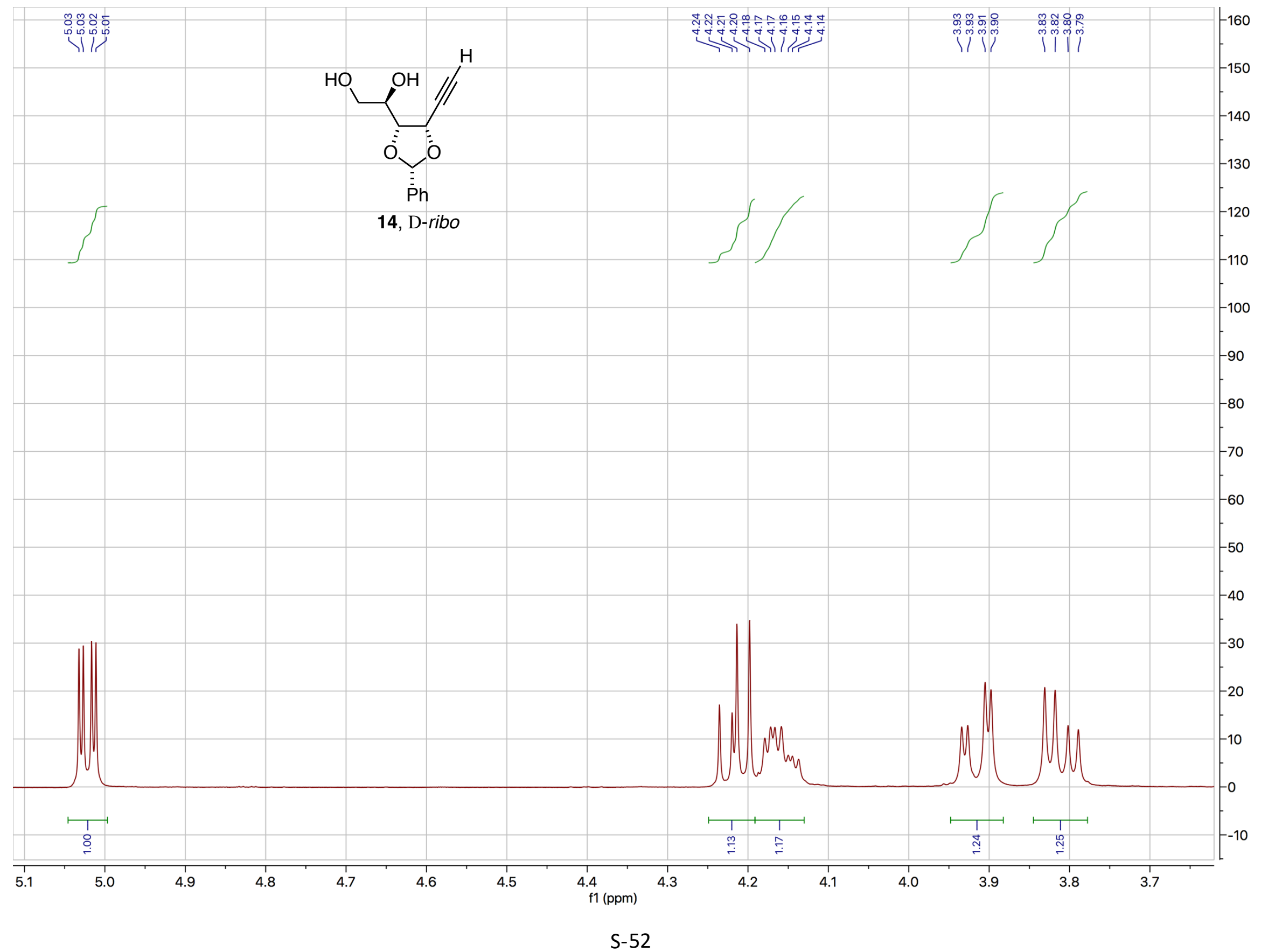


Alkynyl diol 14, ${ }^{13} \mathrm{C}$ NMR (151 MHz, $\left.\mathrm{CDCl}_{3}\right)$

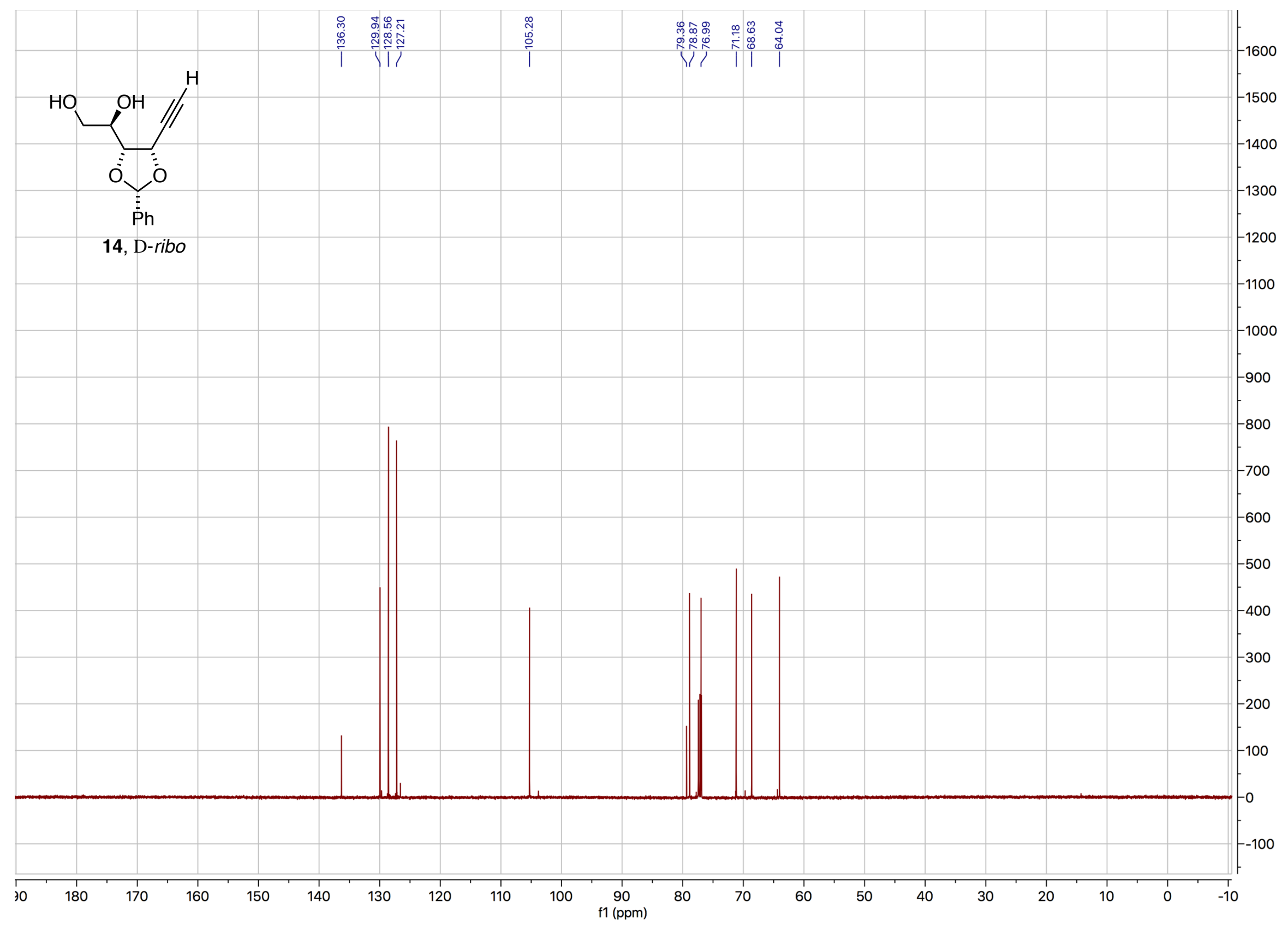


Alkynyl diol 14, $\mathrm{HSQC}\left(\mathrm{CDCl}_{3}\right)$

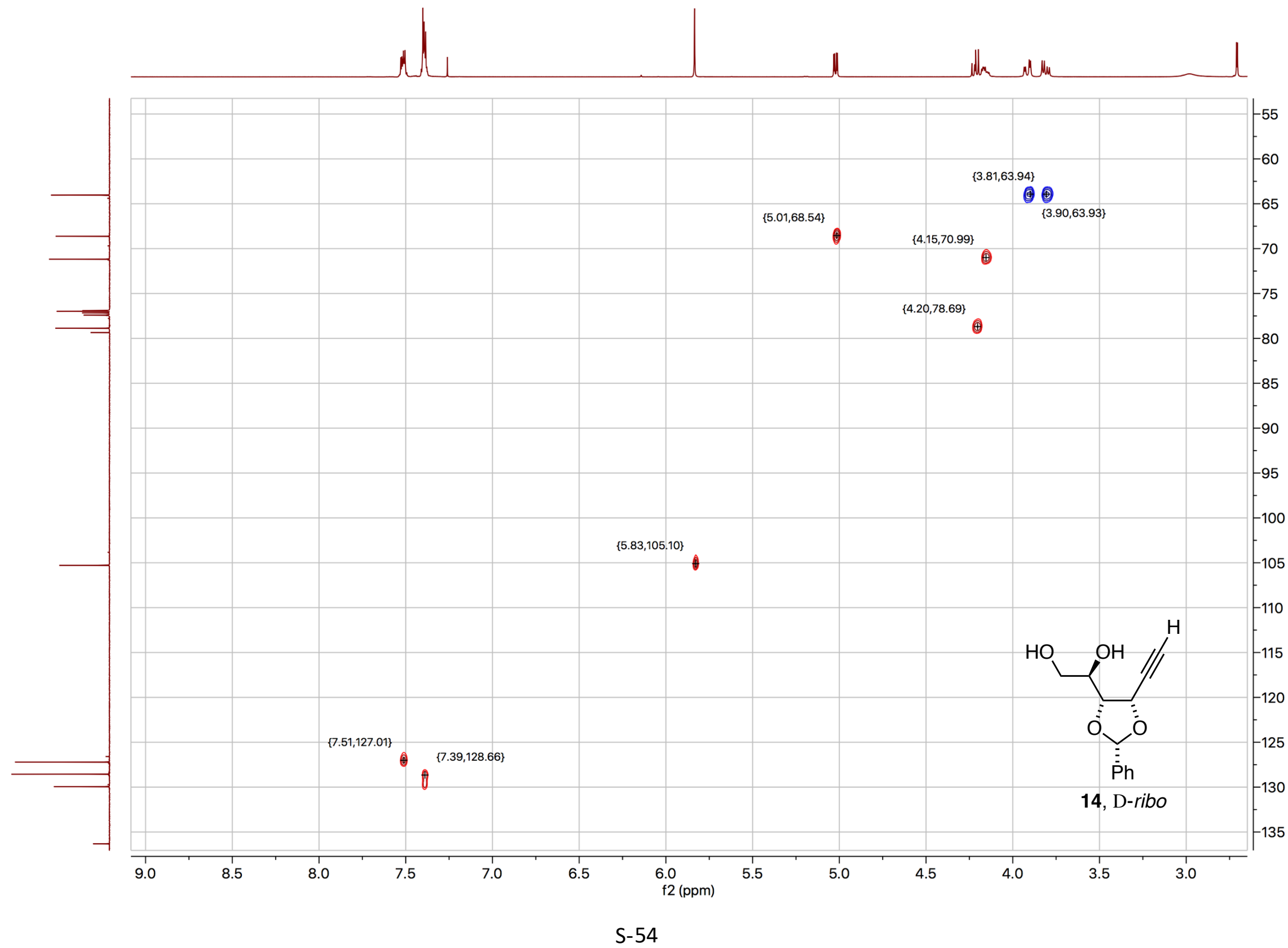


Alkynyl diol 15a-15b mixture, ${ }^{1} \mathrm{H}$ NMR $\left(400 \mathrm{MHz}, \mathrm{CDCl}_{3}\right)$

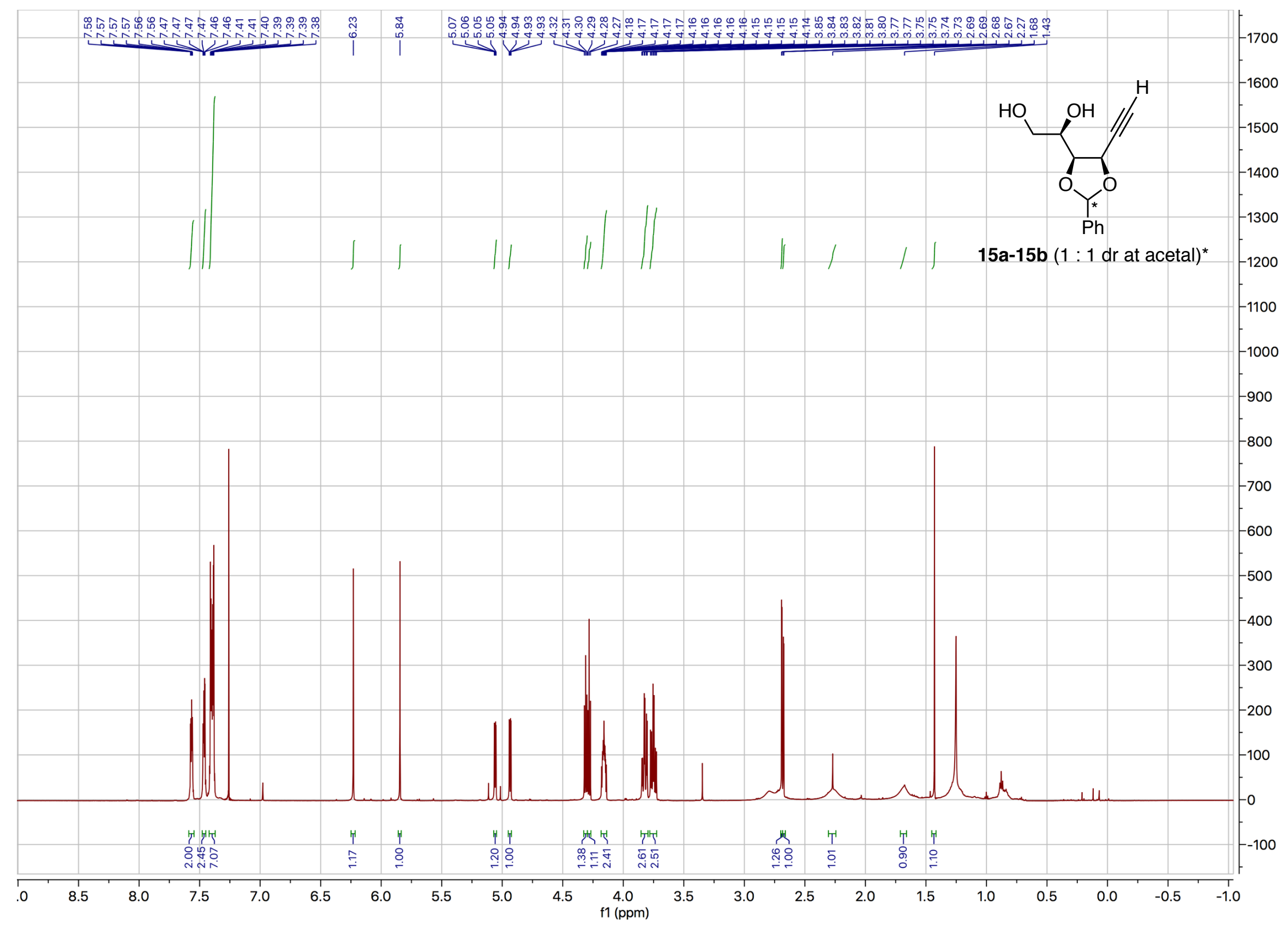

S-55 
Alkynyl diol 15a-15b mixture, ${ }^{1} \mathrm{H}$ NMR (expansion)

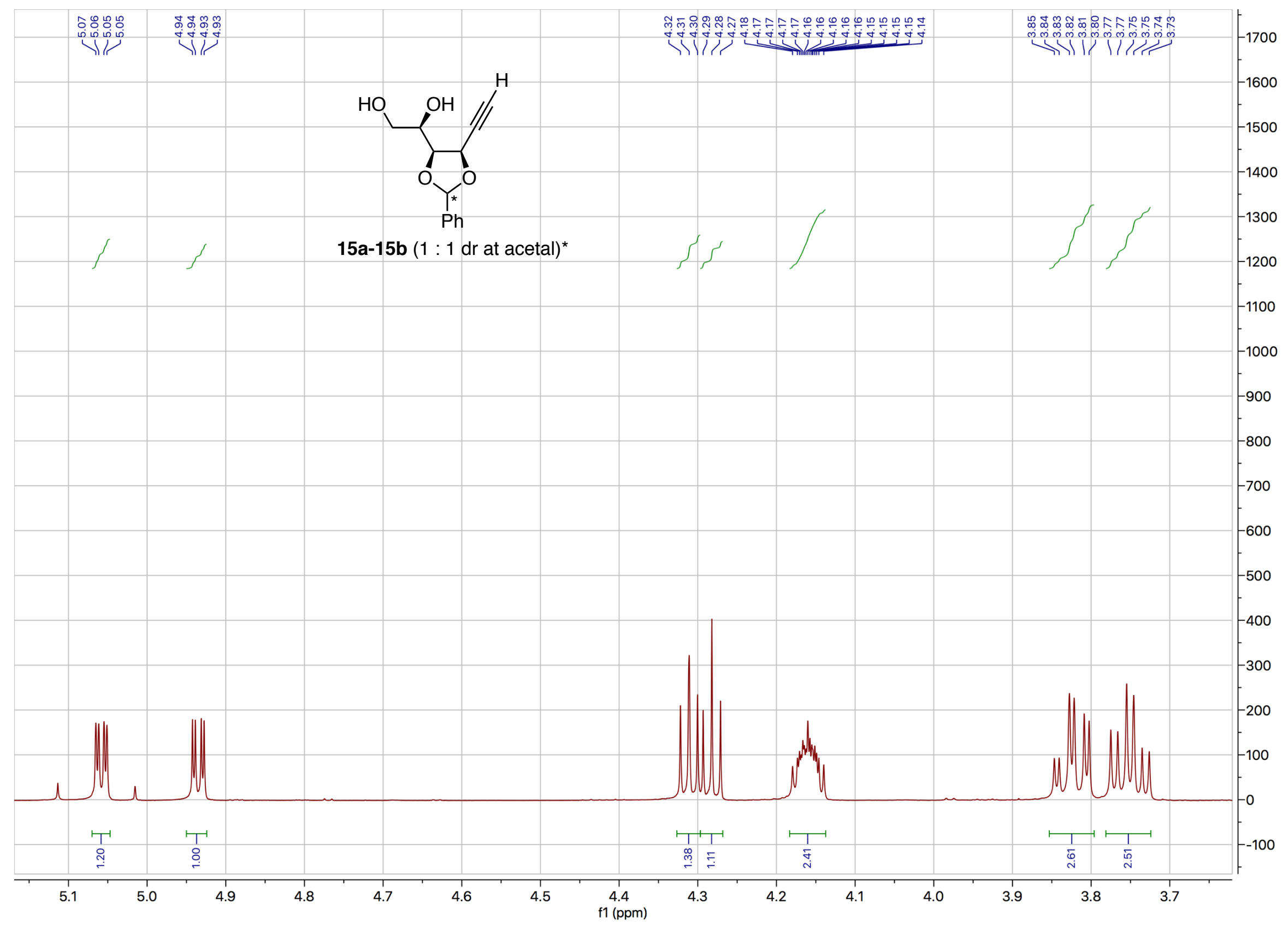


Alkynyl diol 15a, ${ }^{1} \mathrm{H}$ NMR (400 MHz, $\left.\mathrm{CDCl}_{3}\right)$

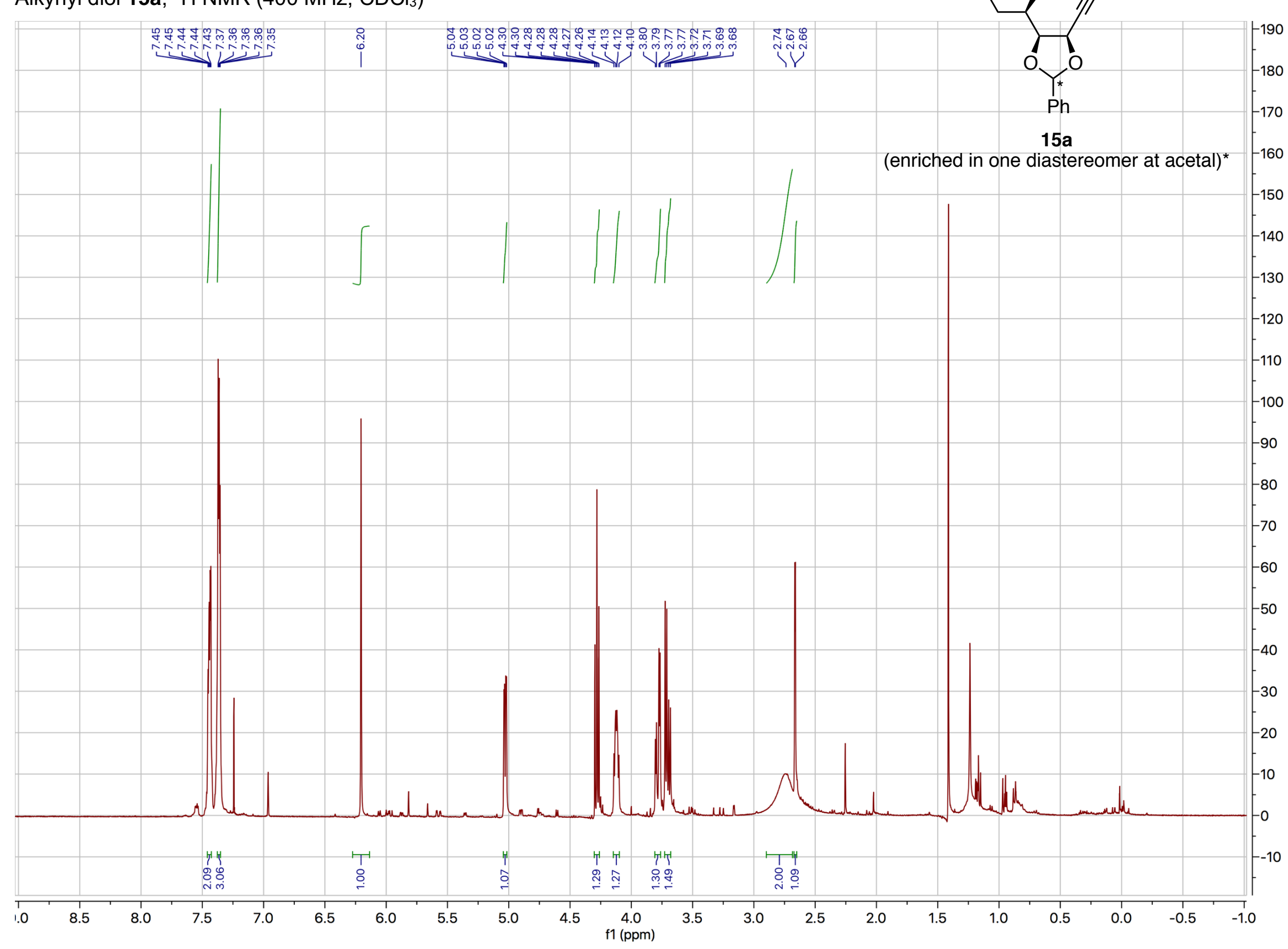

S-57 
Alkynyl diol 15a, ${ }^{1} \mathrm{H}$ NMR (expansion)

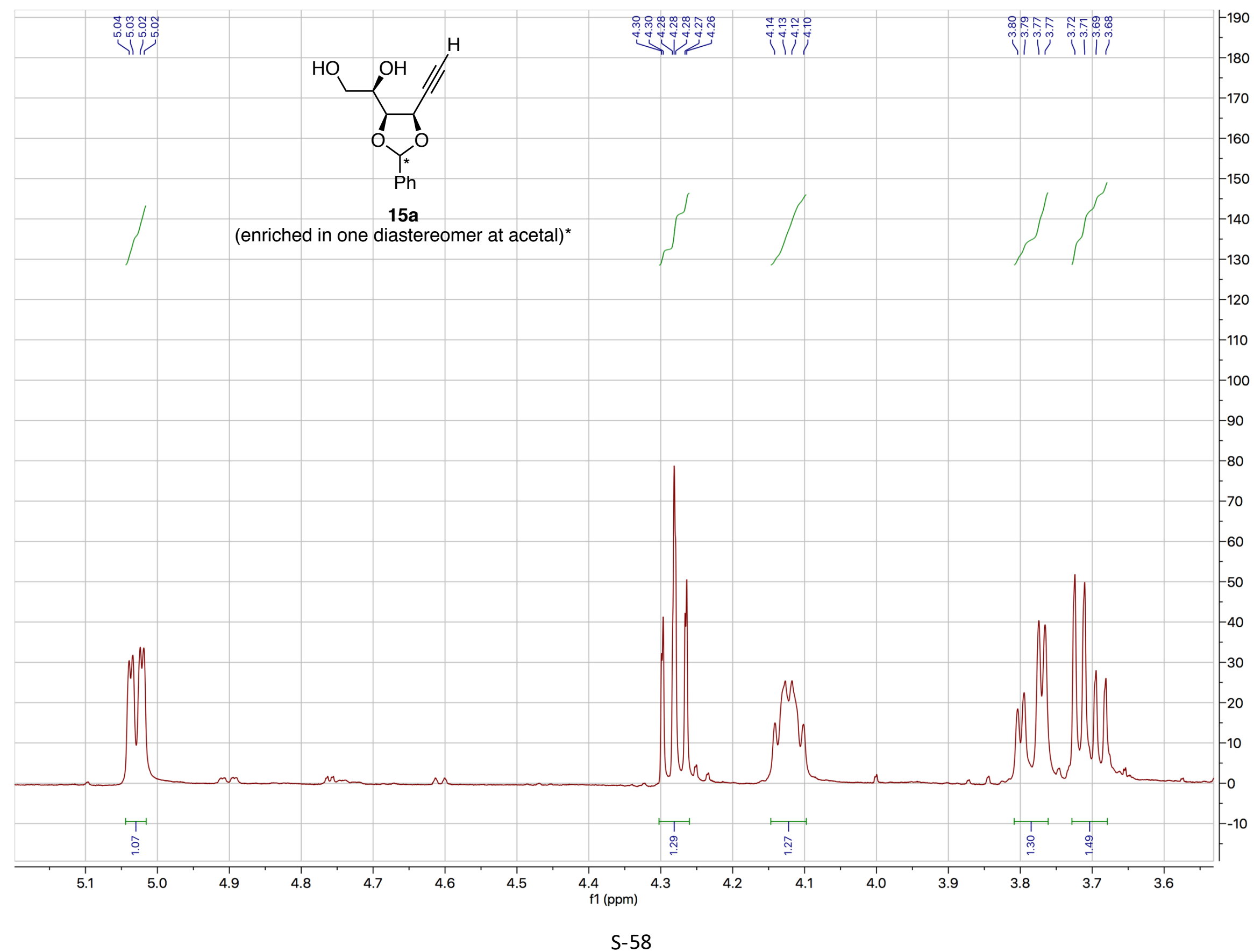


Alkynyl diol 15b, ${ }^{1} \mathrm{H}$ NMR (400 MHz, $\left.\mathrm{CDCl}_{3}\right)$

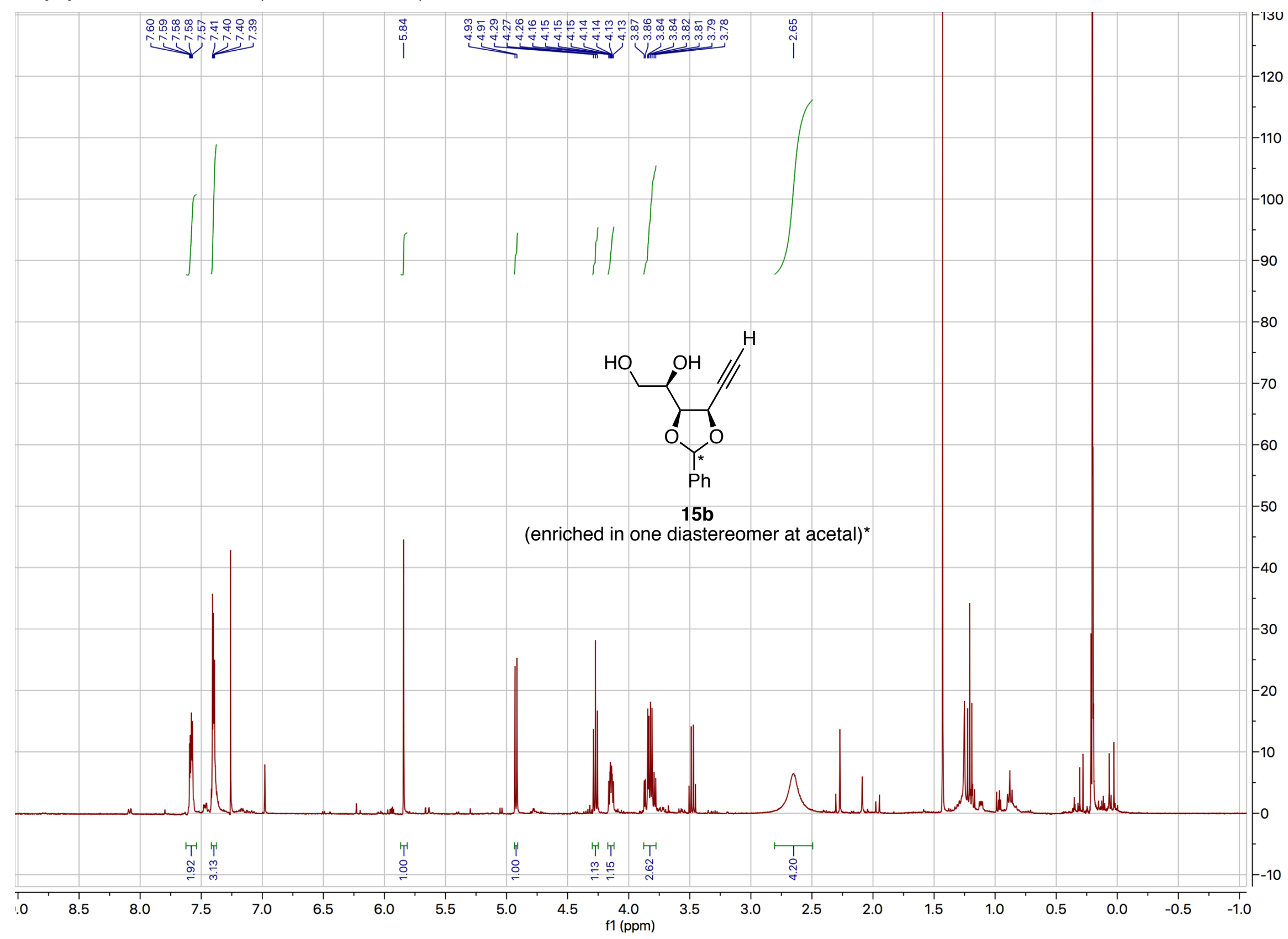


Alkynyl diol 15b, ${ }^{1} \mathrm{H}$ NMR (expansion)

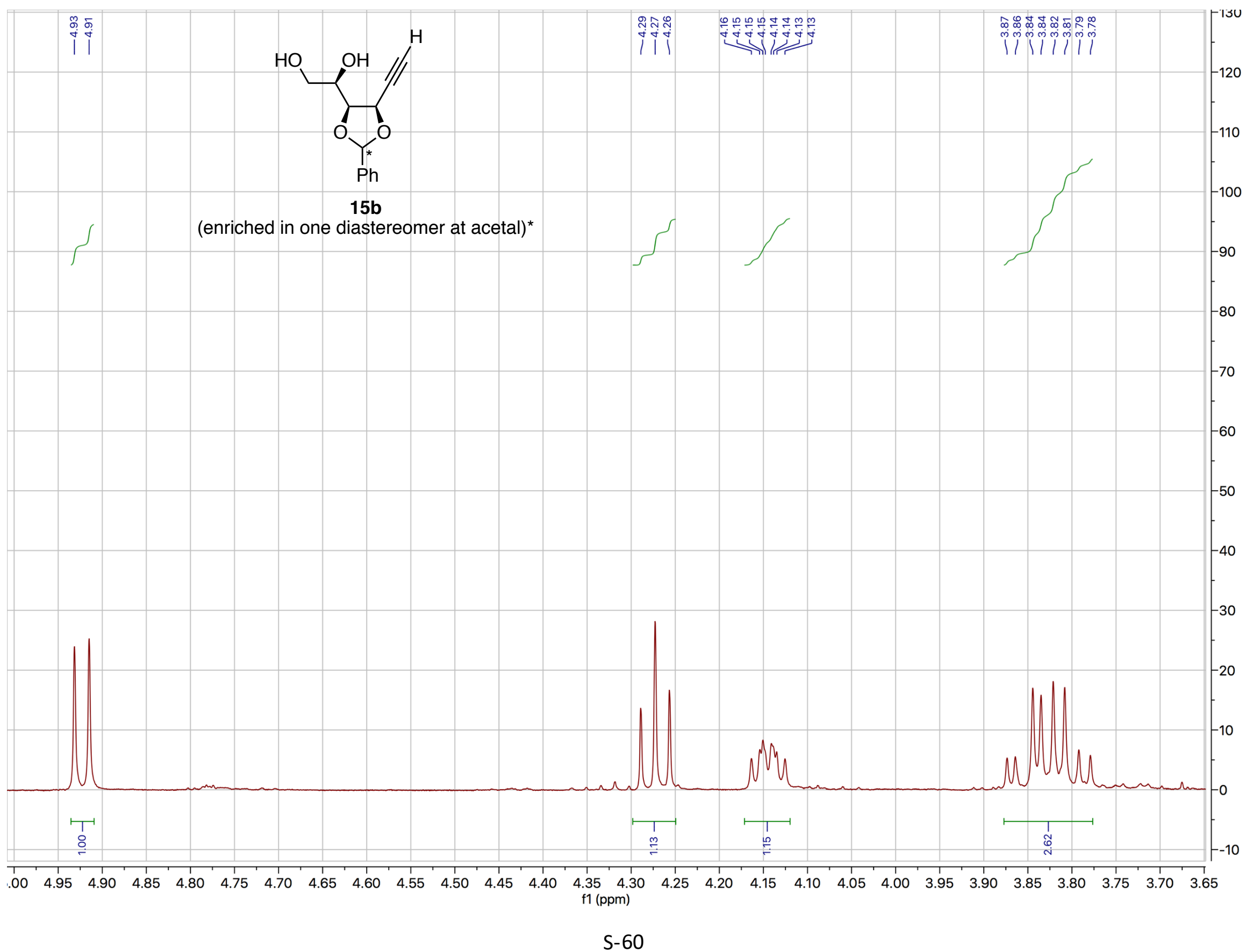


Alkynyl diol 15a-15b mixture, ${ }^{13} \mathrm{C}$ NMR $\left(151 \mathrm{MHz}, \mathrm{CDCl}_{3}\right)$

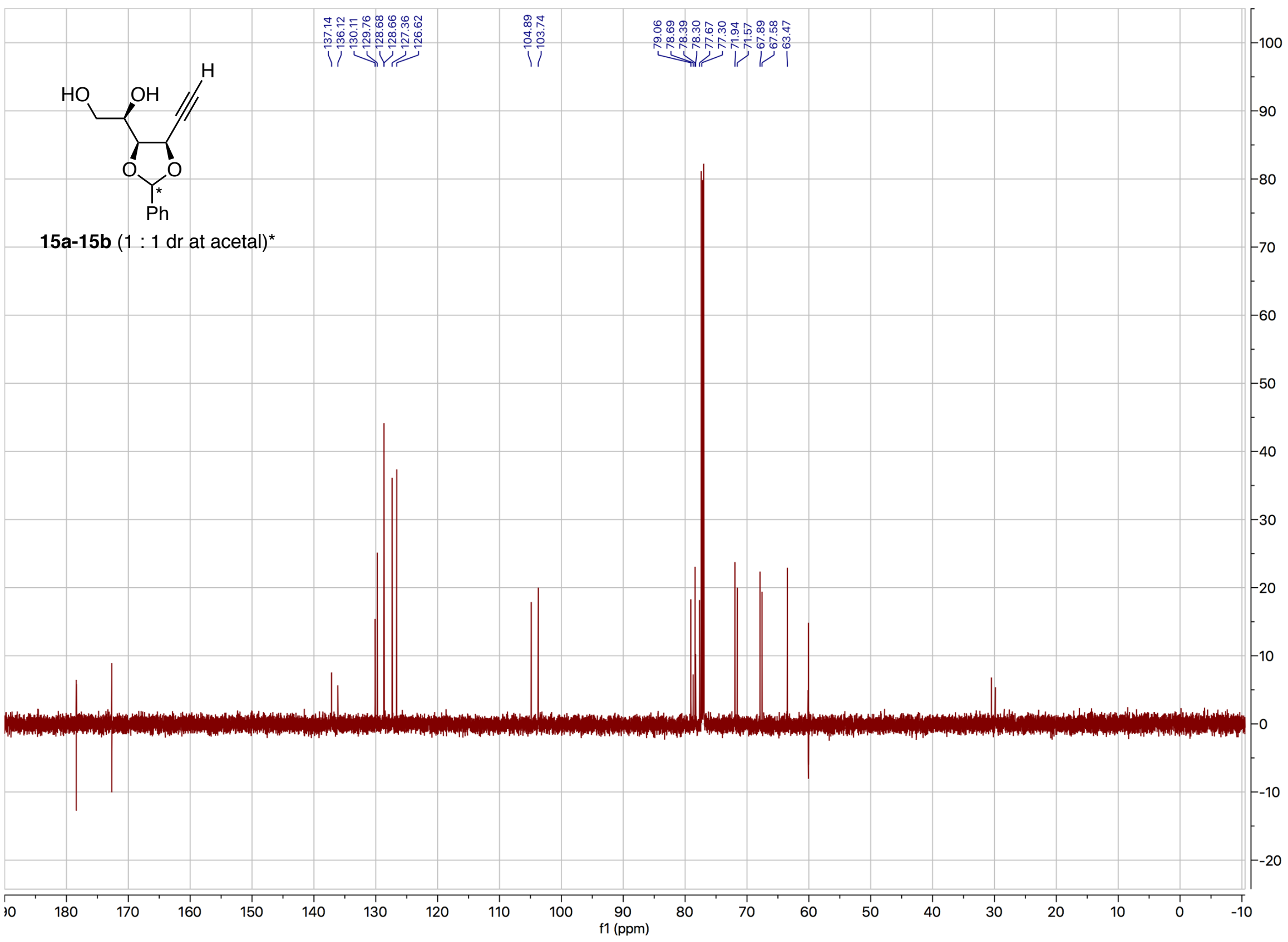

S-61 
Alkynyl diol 15a-15b mixture, HSQC

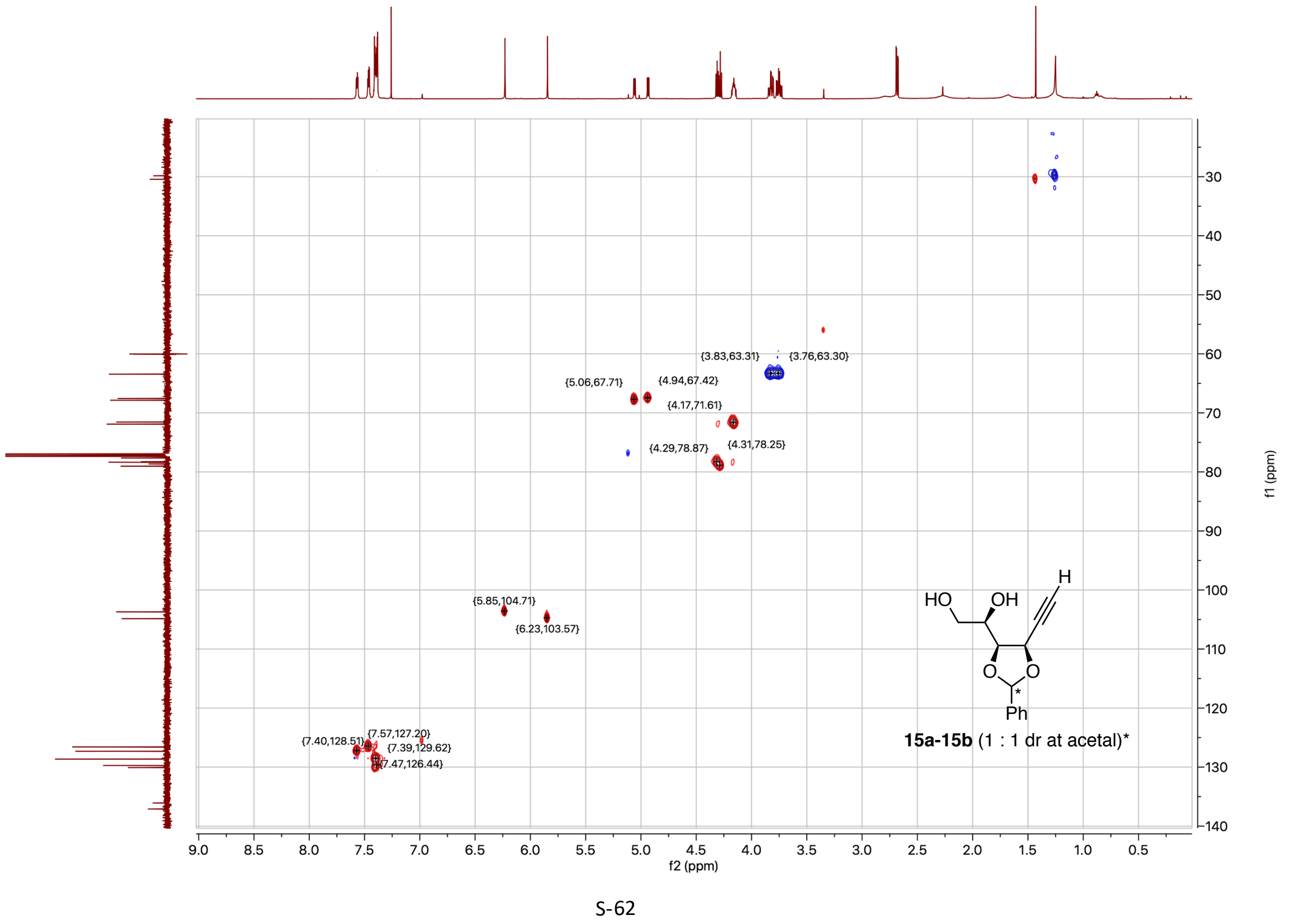


Pyranose glycal 16, ${ }^{1} \mathrm{H}$ NMR $\left(400 \mathrm{MHz}, \mathrm{CDCl}_{3}\right)$

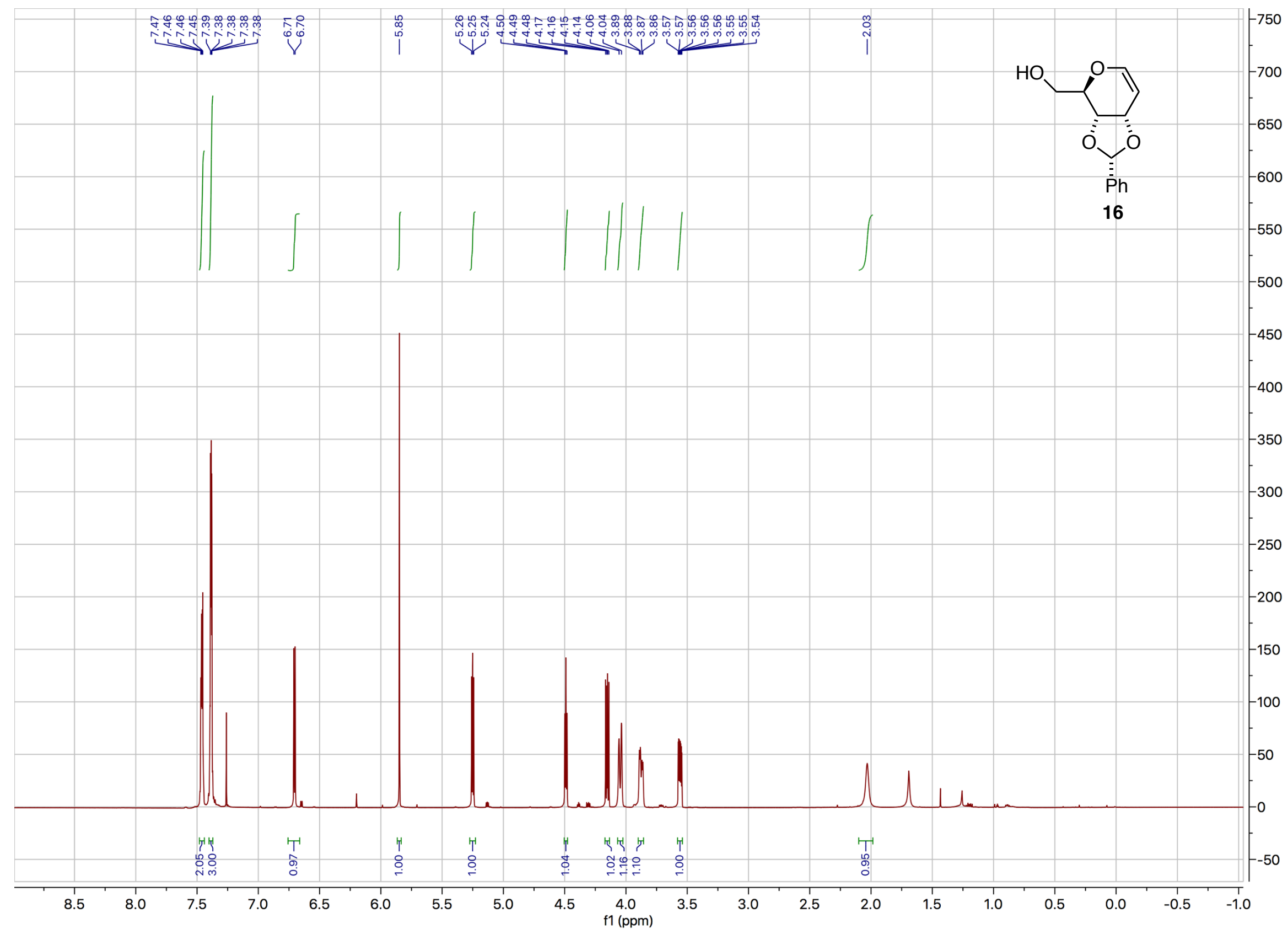


Pyranose glycal 16, ${ }^{13} \mathrm{C}$ NMR $\left(151 \mathrm{MHz}, \mathrm{CDCl}_{3}\right)$

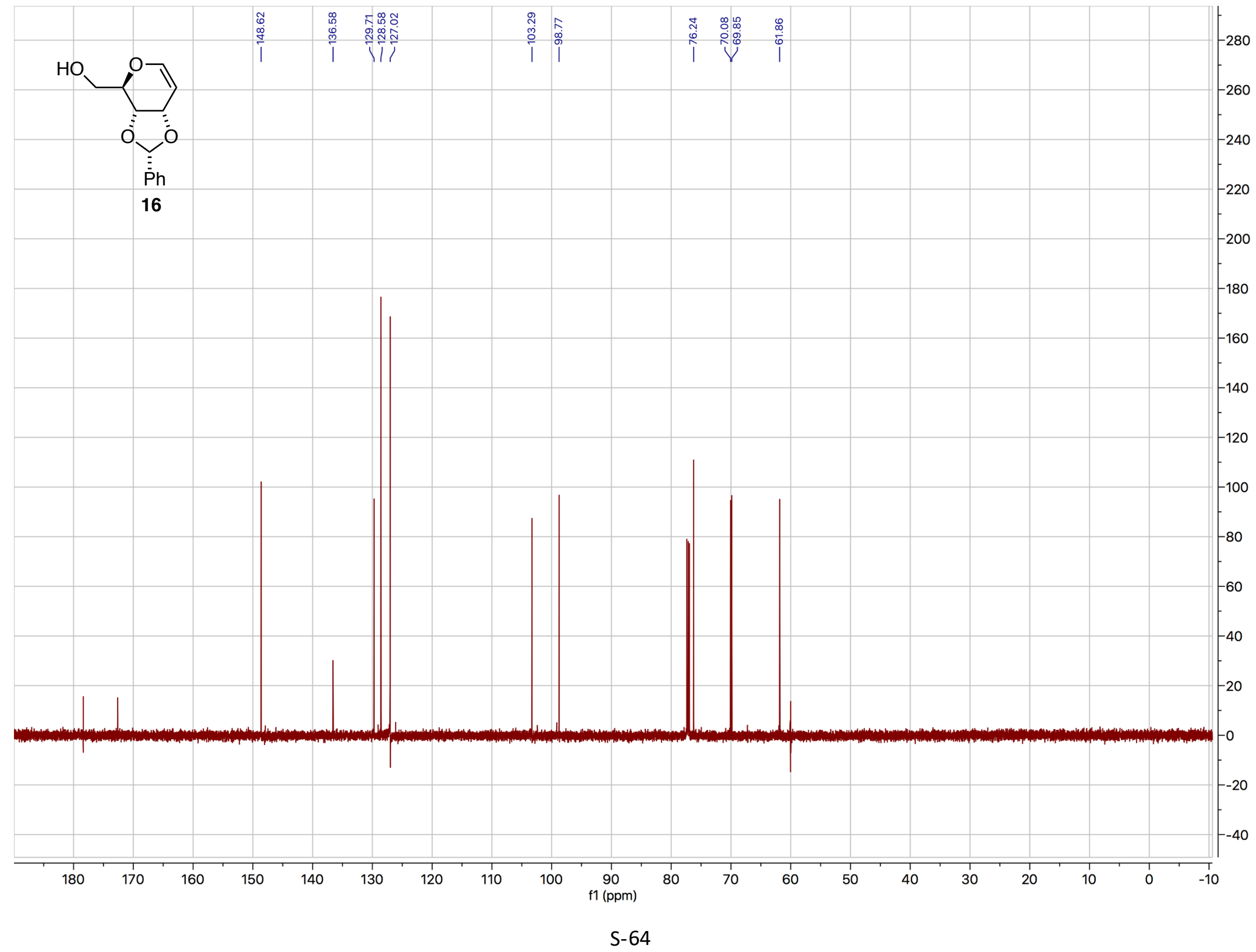


Pyranose glycal 16, HSQC

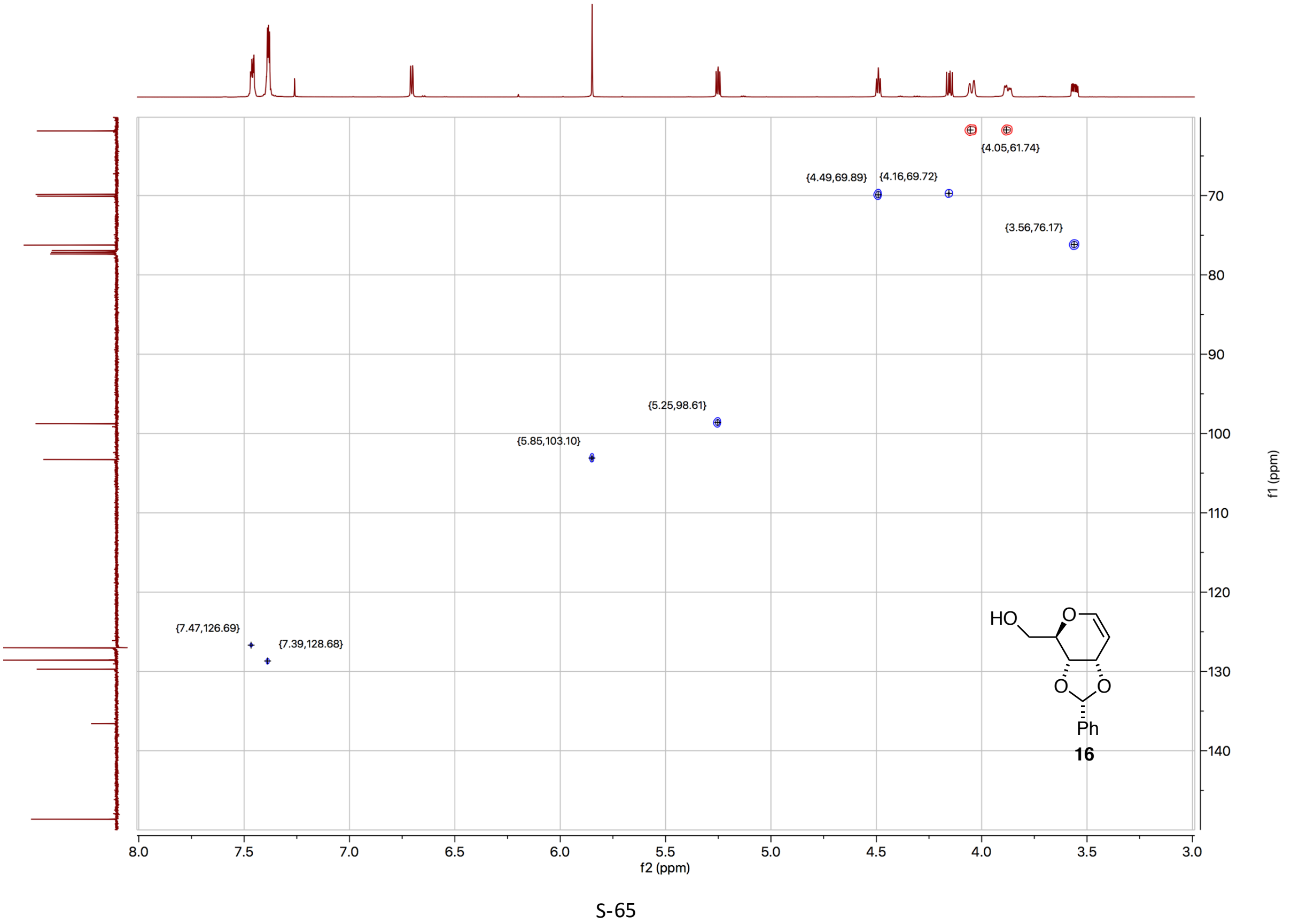


Pyranose glycal 17a, ${ }^{1} \mathrm{H}$ NMR $\left(600 \mathrm{MHz}, \mathrm{CDCl}_{3}\right)$

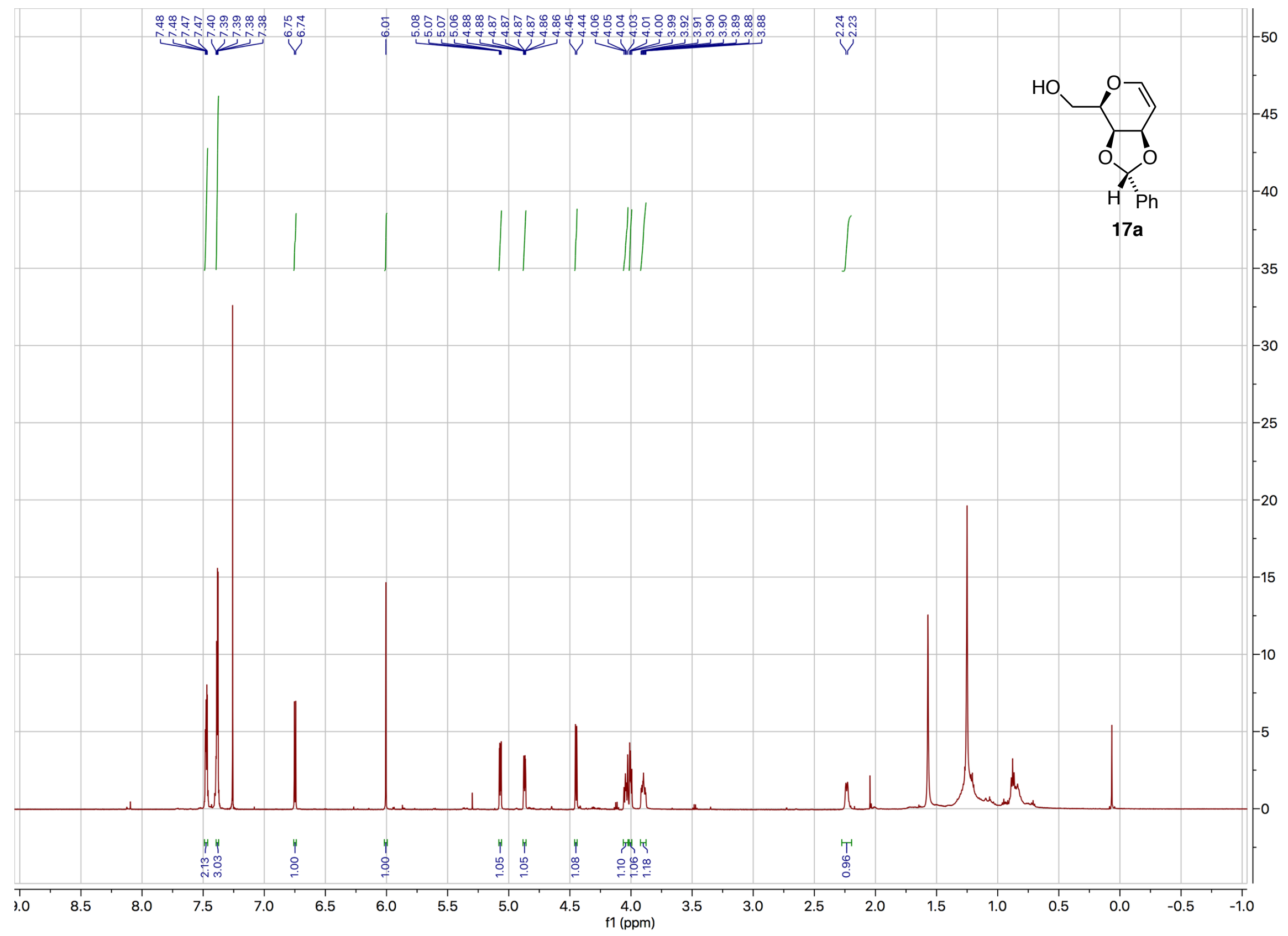

S-66 
Pyranose glycal 17a, ${ }^{13} \mathrm{C}$ NMR (126 MHz, $\left.\mathrm{CDCl}_{3}\right)$

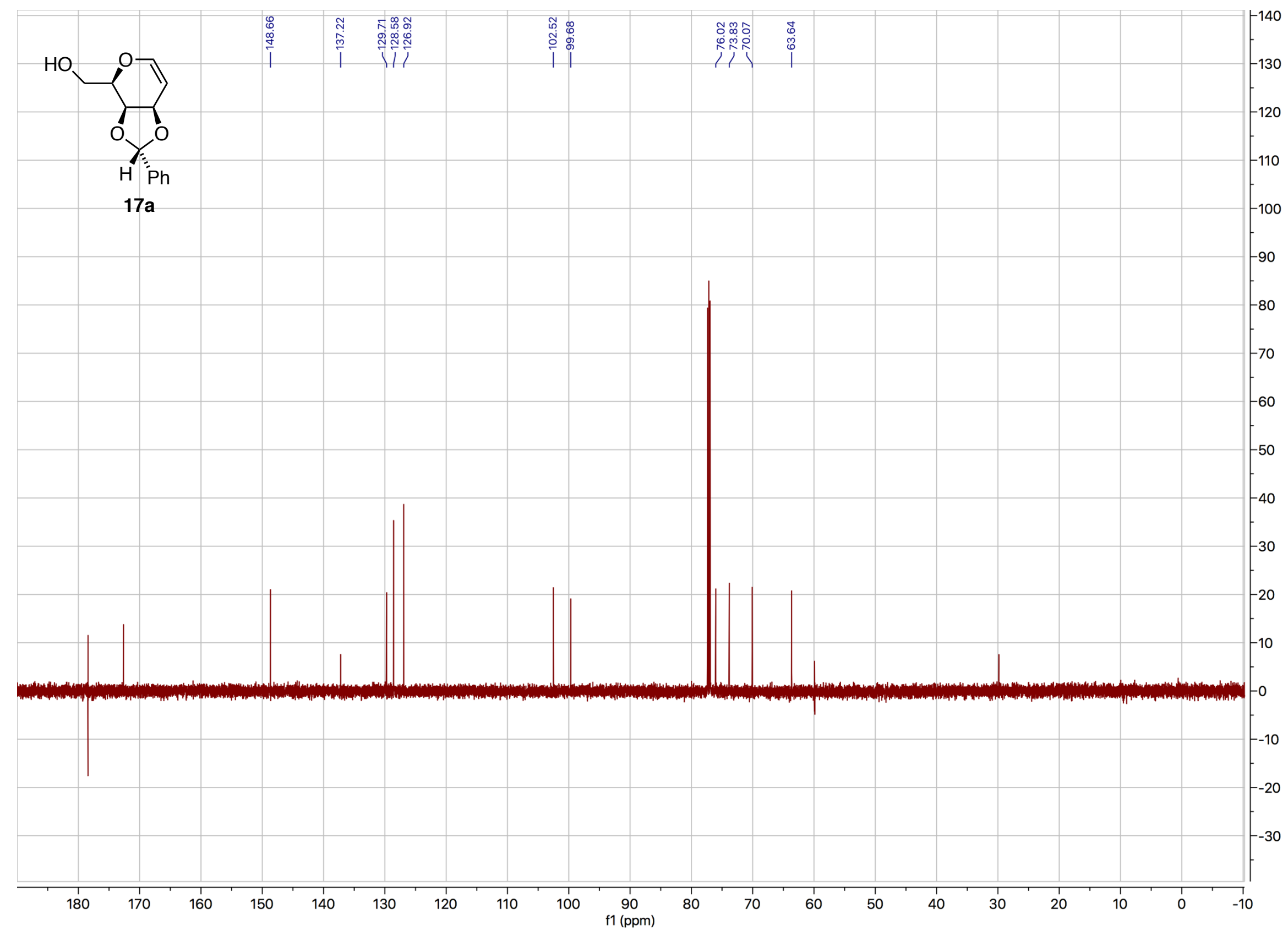


Pyranose glycal 17a, HSQC

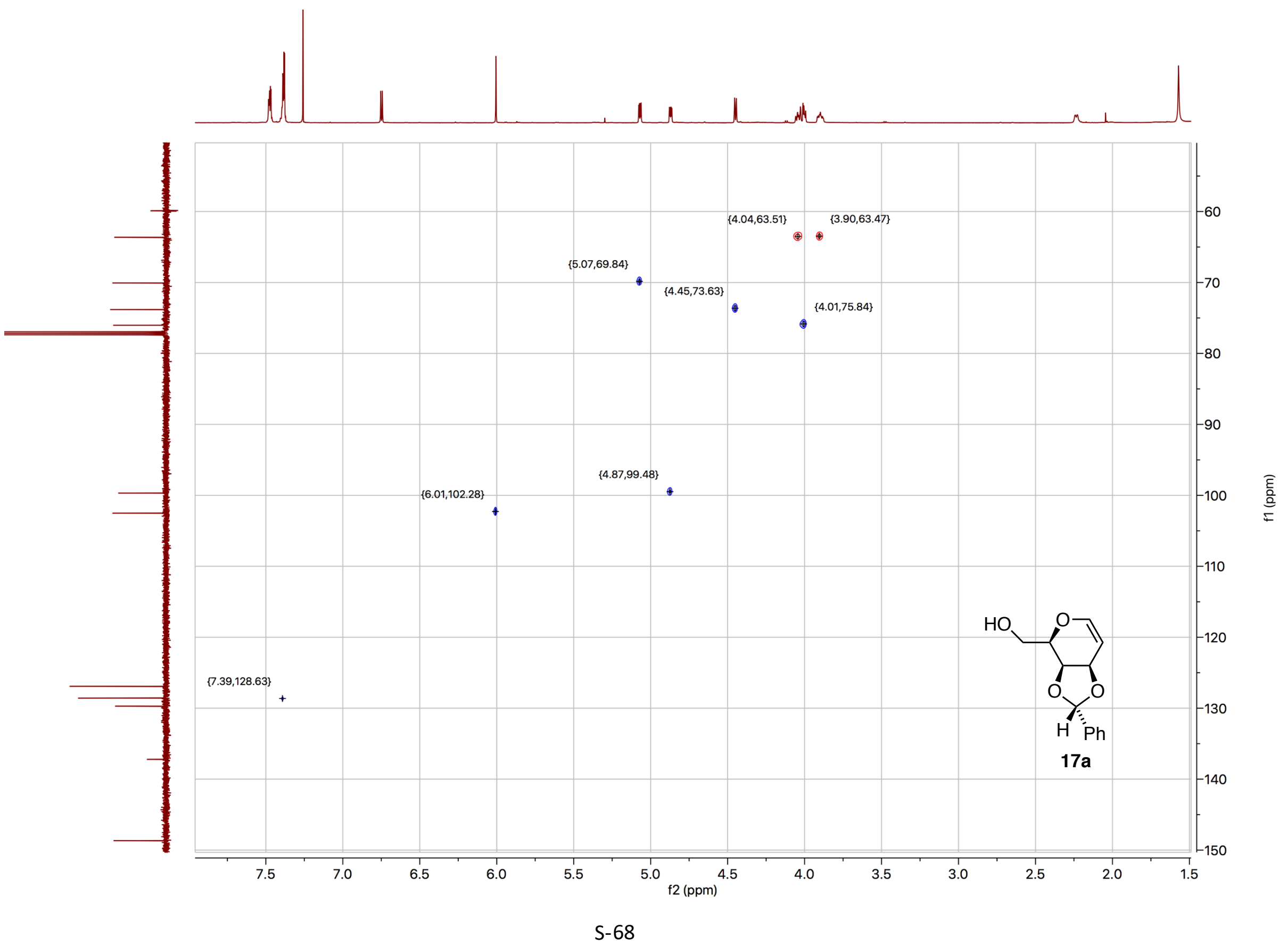


Pyranose glycal 17b, ${ }^{1} \mathrm{H}$ NMR $\left(500 \mathrm{MHz}, \mathrm{CDCl}_{3}\right)$

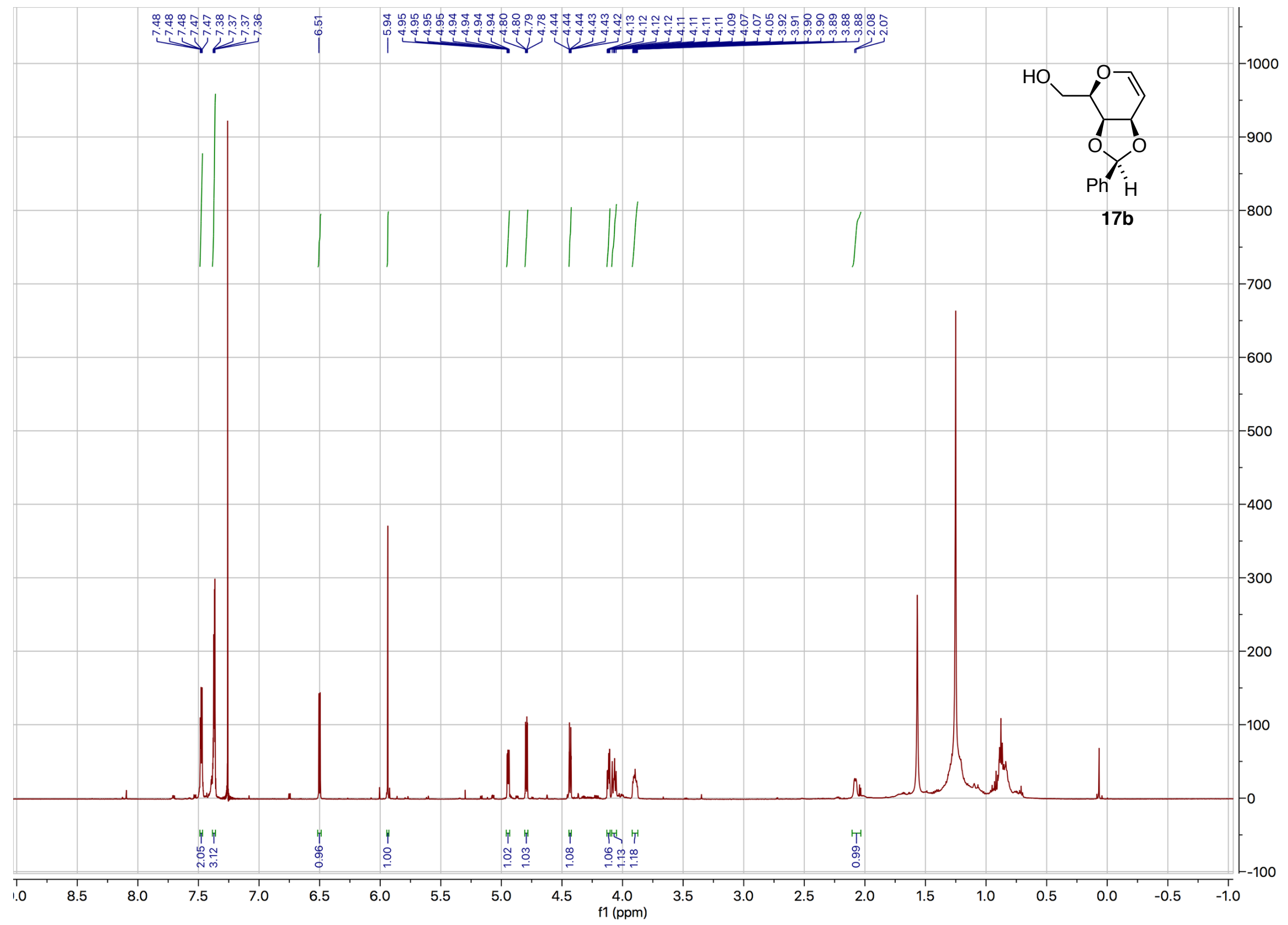

S-69 
Pyranose glycal $\mathbf{1 7 b},{ }^{13} \mathrm{C}$ NMR (151 MHz, $\left.\mathrm{CDCl}_{3}\right)$

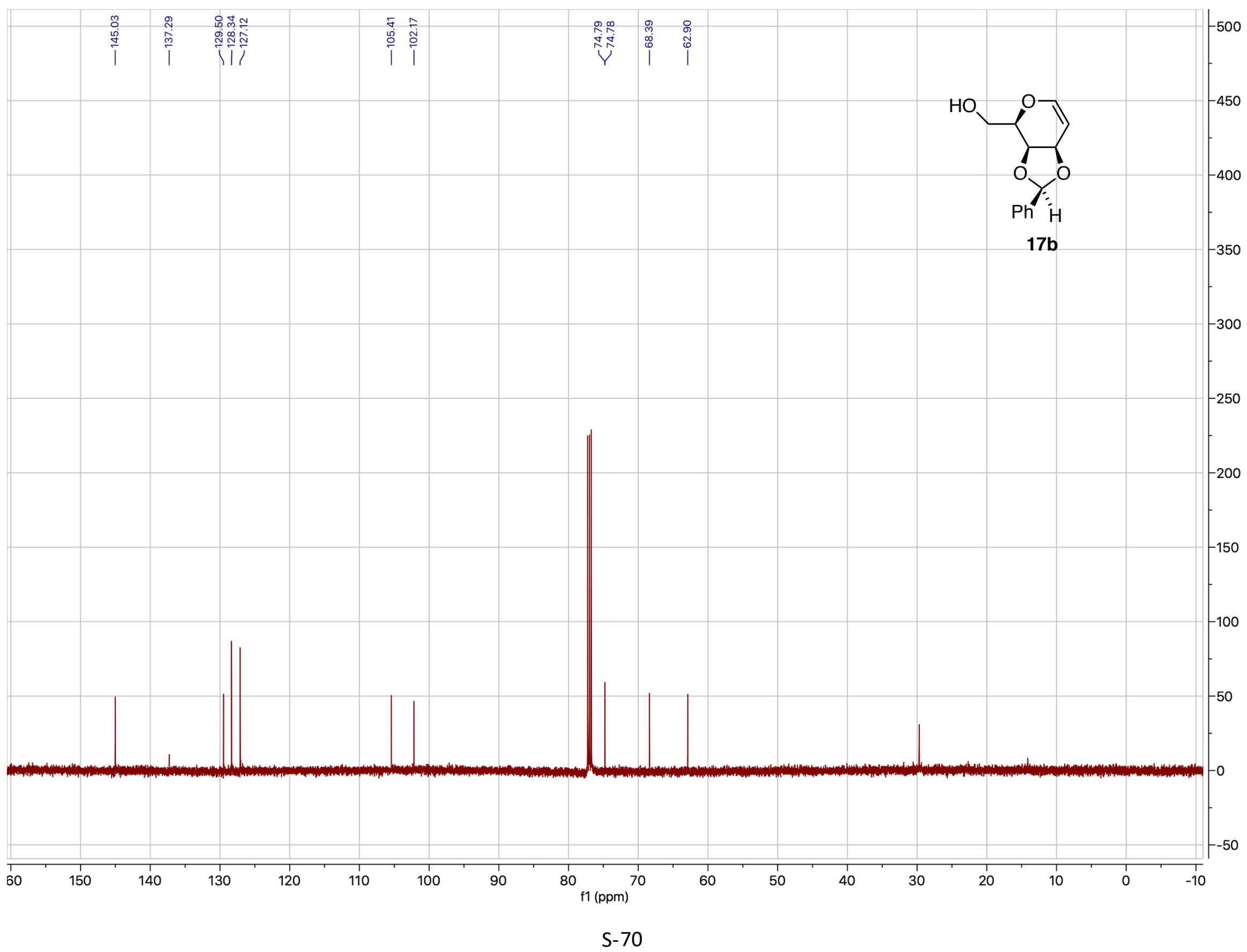

